\title{
Electron Trapping by Polar Molecules in Alkane Liquids: Cluster Chemistry in Dilute Solution.
}

Ilya A. Shkrob and Myran. C. Sauer, Jr.

Radiation and Photochemistry Group, Chemistry Division, Argonne National Laboratory, 9700 South Cass Avenue, Argonne, Illinois 60439

Tel 630-2529516, FAX 630-2524993,e-mail: shkrob@anl.gov.

\section{SUPPLEMENTARY MATERIAL JP050564v \\ Journal of Physical Chemistry A, Received: February 1, 2005 Supporting Information.}

\section{(1.) Appendix: Two-trap model.}

In this Appendix, we consider the model in which two trapped species, electron-1 and electron- 2 exist in thermodynamic equilibrium with a quasifree electron, (Schemes 1 and 2 below). A generalization of this model for more than two trapped species is straightforward.

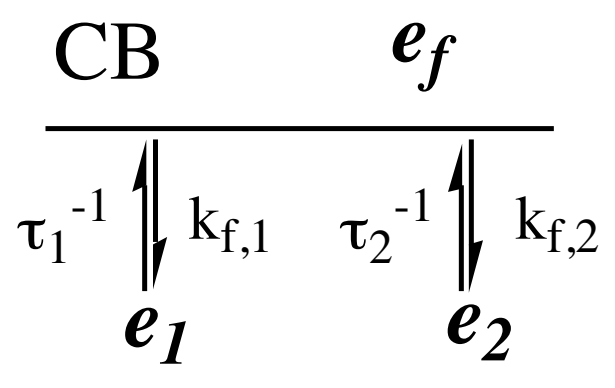

\section{Scheme 1}

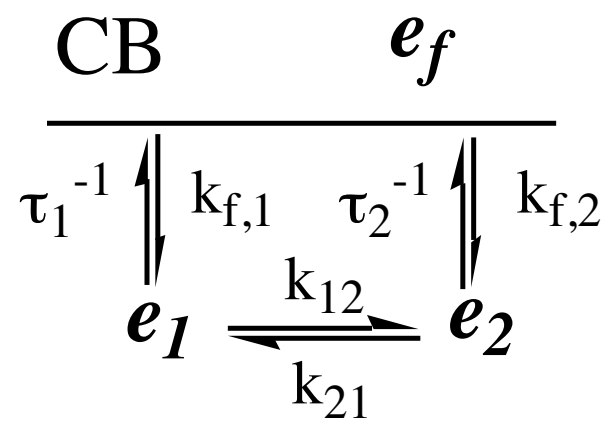

Scheme 2

Let $e_{m}(t)(m=1,2)$ be the concentrations of trapped electrons, $e_{m}^{0}$ be their equilibrium concentrations before photoexcitation promoting the electron to the $\mathrm{CB}, e_{f}(t)$ and $e_{f}^{0}$ be the corresponding concentrations for quasifree electron, $k_{f, m}$ and $k_{m}=\tau_{m}^{-1}$ be the trapping and detrapping rates and $k_{12}$ and $k_{21}$ be the rate constants for direct $1 \rightarrow 2$ and $2 \rightarrow 1$ 
transformations (Scheme 2). We will assume that $k_{m}, k_{12,21}<<\tau_{f}^{-1}=k_{f}=k_{f, 1}+k_{f, 2}$, where $\tau_{f}$ is the lifetime of the quasifree electron, so that $e_{f} \ll e_{m}$ during the photoexcitation. Let

$$
J(t)=\left(J / \tau_{p} \sqrt{\pi}\right) \exp \left(-\left[t / \tau_{p}\right]^{2}\right)
$$

be the irradiance of a Gaussian pulse with a pulse duration $\tau_{p}>\tau_{f}$ and a total fluence $J=\int d t J(t)$ (unless specified otherwise, the integration over time is from $t=-\infty$ to $t=+\infty)$ and $\sigma_{m}$ be the cross sections for electron photodetachment form electron-1 and electron-2 $\left(\sigma_{m} J(t) \ll<k_{f}\right)$. During the photoexcitation pulse, the kinetic equations are given by

$$
\begin{aligned}
& \frac{d e_{1,2}}{d t}=k_{f ; 1,2} e_{f}-k_{1,2}^{\prime}(t) e_{1,2} \mp\left(k_{12} e_{1}-k_{21} e_{2}\right), \\
& \frac{d e_{f}}{d t}=k_{1}^{\prime}(t) e_{1}+k_{2}^{\prime}(t) e_{2}-k_{f} e_{f},
\end{aligned}
$$

where $k_{m}^{\prime}(t)=\sigma_{m} J(t)+k_{m}$. Assuming stationary conditions for $e_{q f}^{-}, d e_{f} / d t \approx 0$ and $e_{f}<e_{1,2}$, we obtain

$$
e_{f}=\tau_{f}\left\{k_{1}^{\prime}(t) e_{1}+k_{2}^{\prime}(t) e_{2}\right\}
$$

and

$$
\frac{d e_{1}}{d t}=-\frac{d e_{2}}{d t}=-\left[P_{2} k_{1}^{\prime}(t)+k_{12}\right] e_{1}+\left[P_{1} k_{2}^{\prime}(t)+k_{21}\right] e_{2},
$$

where $P_{m}=k_{f, m} / k_{f}$ are the partition coefficients $\left(P_{1}+P_{2}=1\right)$ and $e_{1}(t=-\infty)=e_{1}^{0}$. Let us first consider the case where the equilibrium is set before the photoexcitation of the trapped electrons. Since at equilibrium $d e_{m}^{0} / d t=0$, the "equilibrium constant"

$$
K=\frac{e_{2}^{0}}{e_{1}^{0}}=\frac{P_{2} k_{1}+k_{12}}{P_{1} k_{2}+k_{21}} .
$$

Introducing the modified partition coefficients $P_{1}^{\prime}=P_{1}+k_{21} \tau_{2}$ and $P_{2}^{\prime}=P_{2}+k_{12} \tau_{1}$, the latter equation may be written as

$$
K=P_{2}^{\prime} \tau_{2} / P_{1}^{\prime} \tau_{1}
$$


so that

$$
e_{m}^{0} / e_{0}=P_{m}^{\prime} \tau_{m} /\left(P_{1}^{\prime} \tau_{1}+P_{2}^{\prime} \tau_{2}\right)
$$

and

$$
e_{f}^{0} / e_{0}=\tau_{f}\left(P_{1}^{\prime}+P_{2}^{\prime}\right) /\left(P_{1}^{\prime} \tau_{1}+P_{2}^{\prime} \tau_{2}\right)
$$

where $e_{0}=e_{1}+e_{2}$ is the total concentration of negatively charged species. Assuming that only quasifree electrons are conducting (with mobility $\mu_{f}>\mu_{m}$ ), the equilibrium conductivity $\kappa_{0}=F e_{0}\langle\mu\rangle$, where $F$ is the Faraday constant and $\langle\mu\rangle=\mu_{f} e_{f}^{0} / e_{0}$ is the apparent electron mobility given by

$$
\langle\mu\rangle=\mu_{f} \tau_{f} \frac{k_{1} e_{1}^{0}+k_{2} e_{2}^{0}}{e_{0}}=\mu_{f} \tau_{f}\left\langle\tau^{-1}\right\rangle,
$$

where $\langle\ldots\rangle$ stands for averaging over the equilibrium concentrations of traps. We will first consider Scheme 1 in which the direct $1 \leftrightarrow 2$ transformations are neglected. In such a case,

$$
\langle\mu\rangle=\mu_{f} \tau_{f} /\left(P_{1} \tau_{1}+P_{2} \tau_{2}\right)
$$

Let us assume that $e_{1}$ in Scheme 1 is the electron residing in the intrinsic solvent trap $\left(e_{\text {solv }}^{-}\right)$and $e_{2}$ is the electron residing in the solute-associated trap, $\left\{e^{-}: S\right\}_{\text {solv }}$. We further assume that $k_{f, 2}=k_{f, S}[S]$, where $k_{f, S}$ is the second-order constant. Let $\left\langle\mu_{n}\right\rangle=\mu_{f} /\left(k_{f, 1} \tau_{1}\right)$ be the apparent electron mobility in neat solvent. From eq. (A11), we obtain

$$
\left\langle\mu_{n}\right\rangle /\langle\mu\rangle=1+K
$$

where $K=K_{e q}[S]$ and

$$
K_{e q}=k_{f, S} \tau_{2} / k_{f, 1} \tau_{1},
$$

is the equilibrium constant of $1 \leftrightarrow 2$ conversion (eq. (A6) and (A7)). Eq. (A12) is identical with eq. (6) given in the text. Exactly this dependence is given by a two-state model in which one considers a formal equilibrium with the solvent trap $S$

$$
e_{\text {solv }}^{-}+S \underset{\text { 烈 }}{\longleftrightarrow}\left(e^{-}: S\right)_{\text {solv }}
$$


with the equilibrium constant $K_{e q}$, assuming that species $e_{\text {solv }}^{-}$has mobility $\left\langle\mu_{n}\right\rangle$, while $\left(e^{-}: S\right)_{\text {solv }}$ does not contribute to the conductivity signal (note that rate constants of forward and backward reactions (A14) are $P_{2} k_{1}$ and $P_{1} k_{2}$, respectively). We have used such a model both in section 4.1 of this study and in ref. 10 (see section $1 \mathrm{~S}$ therein) without justification; eq. (A12) justifies this approach. If direct $1 \leftrightarrow 2$ transformations (Scheme 2) are involved, assuming that $k_{12}=k^{\prime}[S]$ (i.e., considering eq. (A14) as a real rather than a formal equilibrium reaction), we obtain

$$
\frac{\left\langle\mu_{n}\right\rangle}{\langle\mu\rangle}=\frac{1+K_{e q}[S]+\left(\tau_{2}+K_{e q} \tau_{1}[S]\right)\left(k_{21}+k^{\prime}[S]\right)}{1+k_{21} \tau_{2}+k^{\prime} \tau_{1}[S]},
$$

which gives more complex behavior of the apparent mobility $\langle\mu\rangle$ as a function of $[S]$ than that given by formula (A12), although the deviations are substantial only when reactions (A14) occur on the time scale comparable to $\tau_{1}$ or $\tau_{2}$.

We turn to the behavior of the photoinduced conductivity signal

$$
\Delta \kappa(t)=F e_{0} \mu_{f} \Delta e_{f}(t)
$$

where $\Delta e_{f}(t)=e_{f}(t)-e_{f}^{0}$. The quantity of interest is the ratio $r=\Delta A / \kappa_{0}$, where $\Delta A=\int d t \Delta \kappa(t)$ is the area under the signal. This ratio is given by

$$
r=\int d t \Delta e_{f}(t) / e_{f}^{0} .
$$

For $J \rightarrow 0$, kinetic equations (A5) can be solved perturbatively. Let us assume that $e_{1} \approx e_{1}^{0}+\delta$ and $e_{2}=e_{2}^{0}-\delta$, where $\delta \ll<e_{0}$. Retaining only terms that are linear in $J(t)$ and $\delta$, we obtain

$$
\Delta e_{f} \approx \tau_{f}\left[\left(\sigma_{1} e_{1}^{0}+\sigma_{2} e_{2}^{0}\right) J(t)+\left(k_{1}-k_{2}\right) \delta\right]
$$

and

$$
d \delta / d t \approx-k_{s} \delta+\left(P_{1} \sigma_{2} e_{2}^{0}-P_{2} \sigma_{1} e_{1}^{0}\right) J(t),
$$

where

$$
k_{s}=P_{1} k_{2}+P_{2} k_{1}
$$


is the inverse settling time $\tau_{s}$ of the equilibrium reaction (A14). Solving eq. (A18), we obtain

$$
\Delta e_{f} \approx \tau_{f}\left[\left(\sigma_{1} e_{1}^{0}+\sigma_{2} e_{2}^{0}\right) J(t)+\left(k_{1}-k_{2}\right)\left(P_{1} \sigma_{2} e_{2}^{0}-P_{2} \sigma_{1} e_{1}^{0}\right) \int_{-\infty}^{t} d \tau J(\tau) \exp \left(-k_{s}[t-\tau]\right)\right]
$$

Thus, $\Delta \kappa(t)$ can be represented as a weighted sum of the excitation profile $J(t)$ (the "spike") and the same profile convoluted with an exponential whose time constant equals the settling time of equilibrium reaction (A14) (the "slow" component). The analysis of $\Delta \kappa(t)$ kinetics given in section 4.2.2 is based on this general result. Integrating eq. (A21) from $t=-\infty$ to $t=\infty$ we obtain

$$
\int d t \Delta \kappa(t) \approx J F \mu_{f} \tau_{f}\left[\left(\sigma_{1} e_{1}^{0}+\sigma_{2} e_{2}^{0}\right)+\frac{\left(k_{1}-k_{2}\right)\left(P_{1} \sigma_{2} e_{2}^{0}-P_{2} \sigma_{1} e_{1}^{0}\right)}{P_{1} k_{2}+P_{2} k_{1}}\right],
$$

which can be simplified to

$$
\int d t \Delta \kappa(t) \approx J F \mu_{f} \tau_{f} \frac{\sigma_{1} \tau_{1} e_{1}^{0}+\sigma_{2} \tau_{2} e_{2}^{0}}{P_{1} \tau_{1}+P_{1} \tau_{2}}
$$

Combining eqs. (A11) and (A23), the ratio $r / J$ is expressed as

$$
\left(\frac{r}{J}\right)_{J \rightarrow 0} \approx \frac{\sigma_{1} \tau_{1} e_{1}^{0}+\sigma_{2} \tau_{2} e_{2}^{0}}{e_{0}}=\frac{\sigma_{1} \tau_{1}+\sigma_{2} \tau_{2} K}{1+K} .
$$

The latter can be written as $r \approx\left\langle\sigma_{t} \tau_{t}\right\rangle J$ (eq. (7)) which is correct for multiple trapping (see Appendix A of ref. 6). It can be shown that for Scheme 2, $r \approx\left\langle\sigma \tau^{\prime}\right\rangle J$ where

$$
\tau_{1,2}^{\prime}=\tau_{1,2} \frac{1+\left(k_{12}+k_{21}\right) \tau_{2,1}}{1+k_{12} \tau_{1}+k_{21} \tau_{2}}
$$

With the same assumptions made to derive eq. (A12),

$$
\tau_{s}=\frac{\tau_{2}+\tau_{1} K}{1+K},
$$

i.e., the time constant of the "slow" component (second term in eq. (A21)) approaches $\tau_{2}$ at low $[S](K<<1)$ and $\tau_{1}$ in the opposite limit. Thus, for $\tau_{2} \gg>\tau_{p} \gg>\tau_{1}$ the two-trap model predicts that the $\Delta \kappa(t)$ kinetics exhibits an exponential "tail"; as the solute 
concentration decreases, $\tau_{s}$ increases until it approaches the residence time $\tau_{2}$ of electron-2. The ratio $\xi_{s}$ of areas under the "slow" component and the "spike" is given by the ratio of the second and the first terms of eq. (A22), that is

$$
\xi_{s}=\frac{P_{1} P_{2}\left(\tau_{2}-\tau_{1}\right)\left(\sigma_{2} \tau_{2}-\sigma_{1} \tau_{1}\right)}{\left(\sigma_{1} P_{1} \tau_{1}+\sigma_{2} P_{2} \tau_{2}\right)\left(P_{1} \tau_{1}+P_{2} \tau_{2}\right)}
$$

For $\tau_{2}>\tau_{1}$ and $\sigma_{2} \tau_{2}>>\sigma_{1} \tau_{1}$

$$
\xi_{s} \approx \frac{\left(\tau_{2} / \tau_{1}\right) K}{(1+K)\left(\sigma_{1} / \sigma_{2}+K\right)},
$$

and for $K \ll<1$, the ratio $\xi_{s}$ increases with increasing $K$, whereas for $K>>1, \xi_{s} \propto K^{-1}$ decreases with increasing $K$. The initial increase $\left(\xi_{s} \approx\left(\sigma_{2} \tau_{2} / \sigma_{1} \tau_{1}\right) K\right)$ with increasing $K$ occurs only for $\sigma_{1}>\sigma_{2}$; in the opposite limit $\xi_{s} \approx \tau_{2} / \tau_{1} \gg 1$ (i.e., the "prompt" component is negligible) as soon as $K>>\sigma_{1} / \sigma_{2}$. Provided that $\sigma_{1}>\sigma_{2}$, for low solute concentrations $[S](K<<1)$, the relative weight of the exponential "tail" is low and this weight increases linearly with $[S]$; as the concentration further increases and $K>>1$, this weight decreases and the decay becomes faster. Eventually this decay becomes faster than $\tau_{p}$ and the relative weight also decreases, so the "slow" component cannot be discerned. Though these trends were indeed observed experimentally for $\Delta \kappa(t)$ kinetics obtained in dilute ethanol solutions (section 4.2.2), the overall data cannot be accounted for consistently using the two-trap model. Indeed, for $\sigma_{2}>\sigma_{1}$, the second term in eq. (A21) always prevails and the "prompt" component is not observed for any $K$. This unsettling conclusion refers not only to the case of infinitely small fluence $J$ (which is the only regime that can be handled analytically) but also to arbitrary $J$, as may be shown using numerical simulations. While it is possible, in principle, that for alcohol traps $\sigma_{2} \ll \sigma_{1}$ (due to photostimulated proton transfer competing with electron photodetachment), in such a case the ratio $r$ would stay almost linear with $J$ whereas experimentally the "saturation" (see below) sets in for $J>10^{18}$ photon/ $\mathrm{cm}^{2}$ (section 4.2.2). Our simulations suggest that within the confines of the two-state model, it is simply impossible to obtain both the low-concentration $\Delta \kappa(t)$ curves observed experimentally (that exhibit clearly separable "spike" and "slow" components for $[S] \rightarrow 0$ ) and sigmoid power dependencies. Consequently, any scheme intended to explain these data, even at the qualitative level, has to postulate more than two electron species. 
For arbitrary laser fluence $J(t)$, eq. (A5) must be solved numerically and the ratio $r$ determined using eqs. (A4) and (A17). Since all equations are linear, the result does not depend on $e_{0}$. For Scheme 1, the calculation of the ratio $r$ as a function of $J$ (for known pulse duration $\tau_{p}$ (ca. $4 \mathrm{~ns}$ )) requires the knowledge of five parameters: $\sigma_{1,2}, \tau_{1,2}$, and the equilibrium constant $K$ (eq. (A6)). Two of these parameters, $\sigma_{1} \approx 3.2 \times 10^{-17} \mathrm{~cm}^{2}$ and $\tau_{1} \approx 8.3 \mathrm{ps}$, for electron traps in neat $n$-hexane are known from ref. 24 and our previous study, ${ }^{6}$ respectively. The equilibrium constant $K=K_{e q}[S]$ can be estimated from the $\langle\mu\rangle$ data (section 4.2.1). The product $\sigma_{2} \tau_{2}$ can be estimated from the ratio $r / J$ obtained for $K>>1$ in the low-fluence regime; in this regime (neglecting direct $1 \leftrightarrow 2$ reactions in Scheme 2), $\langle\sigma \tau\rangle \approx \sigma_{2} \tau_{2}$ (ca. $5.6 \times 10^{-26} \mathrm{~cm}^{2} \mathrm{~s}$ for $\left(e^{-}: M e C N\right)_{\text {solv }}$; see section 4.2.1). Thus, the main uncertainty is the cross section $\sigma_{2}$ since the ratio $\sigma_{2} / \sigma_{1}$ is not known (although it can be estimated from the TA experiments within a factor of two). The experimental plots of $r$ vs. $J$ can be simulated using this approach with minimal adjustments to the model parameters. Since no refinement of the model parameters can be made using such simulations, the main goal of these calculations is to demonstrate that the trends observed experimentally in Figs. 11S can be rationalized using the two-trap model of Scheme 1; the exact choice of simulation parameters is not important for such a demonstration.

Fig. 21S(a) shows a family of $r$ vs. $J$ plots for different values of the equilibrium constant $K$. Since $\sigma_{2} \tau_{2}>\sigma_{1} \tau_{1}$, even for relatively low values of $K$ the initial slope of these plots is close to $\sigma_{2} \tau_{2}$. For higher fluence, the ratio "saturates". As seen from the plots, for higher $K$ this saturation is less expressed (see also the normalized plots in Fig. $21 \mathrm{~S}(\mathrm{~b})$ illustrating the decreases in the curvature), because the equilibrium can be continuously shifted within the duration of the pulse. Exactly these patterns are observed experimentally (Fig. 11S and 20S). Plotted as a function of the equilibrium constant $K$ for a given fluence $J$, the ratio $r$ rapidly reaches a plateau at lower fluence (as follows from eq. (A24)) but continues to increase with increasing $K$ at greater fluence (Fig. 22S). Once more, the same behavior was observed in dilute acetonitrile solutions in $n$-hexane (Fig. 4).

When direct $1 \leftrightarrow 2$ reactions are introduced (Scheme 2), the bicomponent kinetics can be readily obtained even for $\sigma_{2}>\sigma_{1}$. It may seem that the inclusion of these additional reactions violates the detailed equilibrium principle which relates the equilibrium constant of the $1 \leftrightarrow 2$ reaction with the equilibrium constant for reaction (A14). However, it should be kept in mind that Scheme 1 does not make any provision for interconversion of electron traps (such as reaction (5) in the text) occurring separately from reaction (A14). It is precisely the principle of detail equilibrium that allows one to treat this reaction as an additional equilibrium involving filled traps. 


\section{(2.) Section 1S: Irreversible trapping.}

The conductivity measurements presented in section 4.2 are not sensitive to electrons that are irreversibly trapped by higher multimers (for which the thermal detrapping time is longer than electron lifetime). Still, the electron could be photodetached even from such a species provided that the latter absorbs laser light. ${ }^{6}$ An additional $\Delta \kappa$ signal with the amplitude increasing with the delay time $t_{L}{ }^{6}$ would be expected from such a photoexcitation. The time profile of the resulting $\Delta \kappa$ signal would be identical with that of $\kappa(t)$, save for the delay time of the $1064 \mathrm{~nm}$ pulse. ${ }^{6,10}$ Surprisingly, no such additional signal was observed in either photosystem. Since these stable $\left\{e^{-}: S_{n}\right\}_{\text {solv }}$ species should absorb in the near infrared (as suggested by TA spectra in section 4.1) we are forced to conclude that the electron cannot be detached from large ethanol clusters by $1064 \mathrm{~nm}$ photons. As the TA signals observed in pulse radiolysis experiments decay in $1 \mu \mathrm{s}$, it is possible that electrons in such clusters are unstable, decaying by proton transfer to $R O^{-}$anions, as happens for $e_{\text {solv }}^{-}$in neat alcohols. ${ }^{53,55}$ This slow reaction, however does not explain the lack of $\Delta \kappa$ signal from such a stable $\left\{e^{-}: S_{n}\right\}_{\text {solv }}$ species at shorter delay times (50-200 ns). Thus, we are left with the conclusion that the quantum yield for photodetachment from such clusters must be very low, either due to the predominance of bound-to-bound $(s \rightarrow p)$ transitions and/or to rapid proton transfer in the excited state (as occurs in neat alcohols). ${ }^{55}$

For acetonitrile in $n$-hexane, a similar problem exists since no conclusive evidence for IR-absorbing $\left\{e^{-}:[\mathrm{MeCN}]_{n}\right\}_{\text {solv }}$ multimer species was found in our photodetachment experiments, though there is some evidence for such a species (or a molecular anion) in concentrated solutions in iso-octane (section 4.2.1). The possible explanation for inefficient "dipole coagulation" is that the reaction of $\left\{e^{-}: M e C N\right\}_{\text {solv }}$ with acetonitrile yields the dimer anion, $(\mathrm{MeCN})_{2}^{-} .{ }^{10}$ This covalently-bound anion absorbs poorly in the infrared, as its absorption band is centered in the visible. ${ }^{10,11,14}$ Upon excitation in the vis and infrared, it photodissociates to $\mathrm{CH}_{3}+\mathrm{CN}^{-}+\mathrm{MeCN}$, although for $1064 \mathrm{~nm}$ photoexcitation the quantum yield is very low $(<0.01) .{ }^{10}$ The increase in the TA signal in the visible at longer delay time observed in pulse radiolysis experiments (Figs. 4S and 6S) can be accounted for by the slow generation of dimer anion via reaction (5), although a persistent cation (sections 2.2 and 4.1) can also explain these observations. The photodetachment experiments using $532 \mathrm{~nm}$ light ${ }^{10}$ indicate that shorter-wave photoexcitation does induce the expected long-lived $\Delta \kappa$ signal with a pattern typical of a molecular anion or a stable $\left\{e^{-}:[\mathrm{MeCN}]_{n}\right\}_{\text {solv }}$ species (see Figs. 7S and $8 \mathrm{~S}$ in the Supplement of ref. 10). The results for iso-octane suggest that electron 
(C) 2005 American Chemical Society, J. Phys. Chem. A, Shkrob jp050564v, Supp. Info p. 9

trapping by such a species may in fact be reversible; however, reaction (5) is strongly shifted towards the anion in the concentration range where eq. (6) does not hold.

9S. 


\section{Figure captions (1S to 22S).}

\section{Fig. 1S.}

Speciation plots for neutral alcohol clusters $S_{n}$ in $n$-hexane using the thermodynamic data from ref. 28 and eq. (10) for (a) $23{ }^{\circ} \mathrm{C}$ and (b) $1.8{ }^{\circ} \mathrm{C}$. The net alcohol concentration is given as mole fraction $\chi$ of the alcohol. The monomer fraction (dashed line) is given to the right; equilibrium fractions $f_{n}=n\left[S_{n}\right] / c$ (solid lines) are plotted to the left (the cluster numbers $n$ are given in the legends).

\section{Fig. 2S.}

(a) TA spectra from pulse radiolysed Ar-saturated (open symbols) and $\mathrm{CO}_{2}$-saturated (trace (i), filled circles) room temperature $n$-hexane. $\mathrm{CO}_{2}$ is added as electron scavenger. The integration time windows are given in the plot, for trace (i), the integration window is 6 to $10 \mathrm{~ns}$ (the absorbance spectrum, which is mainly from the solvent olefin cation does not evolve in the first $500 \mathrm{~ns}$ after the electron pulse). This trace was normalized at $500 \mathrm{~nm}$, where most absorbance is from the cation. In Ar-saturated $n$-hexane the absorbance is composite: both $e_{\text {solv }}^{-}$and the cation contribute to the spectrum at short delay times. At later delay times, the relative contribution from the cation increases as the electron is scavenged by impurity. (b) Decay kinetics of optical absorbance at 0.5 and $1.55 \mu \mathrm{m}$ in Ar- and $\mathrm{CO}_{2}$-saturated solutions (see the legend in the plot). Only electrons absorb at the longer wavelength, while at the shorter wavelength, only the cations absorb. Upon the addition of electron scavenger, the end-of-the pulse yield of cations increases ca. 2 times. The smooth (black) line drawn through the $1.55 \mu \mathrm{m}$ curve is single exponential fit (illustrating that electrons mainly decay via scavenging by an impurity).

\section{Fig. 3S.}

TA kinetics $(\lambda=1 \mu \mathrm{m})$ from Ar-saturated $n$-hexane solutions at $23{ }^{\circ} \mathrm{C}$ containing (a) acetonitrile and (b) ethanol. (Same conditions as in Fig. 2S). The net solute concentrations are indicated in the plots. The dashed trace in (b) is the scaled TA kinetics in neat $n$-hexane (drawn to illustrate slowing down of the decay kinetics in ethanol solutions).

\section{Fig. 4S.}

Normalized TA spectra from Ar-saturated acetonitrile/n-hexane solutions $\left(23{ }^{\circ} \mathrm{C}\right.$, the same radiolysis conditions as in Fig. 2S). The net molar concentration of the solute is given in (a). The integration window is (a) 11 to $17 \mathrm{~ns}$ and (b) 80 to $180 \mathrm{~ns}$. The lower the $\mathrm{MeCN}$ concentration the less is the signal from olefin cations in the visible at later delay times. To facilitate the comparison the spectra were normalized at $1.55 \mu \mathrm{m}$, where only electron contributes to the absorbance.

\section{Fig. 5S.}


Normalized TA kinetics (b) and end-of-pulse electron absorbances (a) for $\lambda=1.55 \mu \mathrm{m}$ in room temperature solutions of acetonitrile in iso-octane (the concentrations are given in (b)). The bold line in (a) is the linear plot.

Fig. 6S.

(a-c) Time evolution of absorbance spectra observed in pulse radiolysis of room temperature Ar-saturated, acetonitrile/iso-octane solutions (the concentrations are given in (a)). The spectra are normalized at $1.55 \mu \mathrm{m}$, where only trapped electron absorbs the analyzing light. At short delay times, the spectrum is dominated by this trapped electron; at later delay times the signal from an olefin cation (or a dimer anion) can also be observed.

\section{Fig. 7S.}

A plot illustrating lack of time evolution for absorbance spectra observed in pulse radiolysis of ethanol $/ n$-hexane solutions (the time windows are given in the plots). The spectra obtained for (a) $121 \mathrm{mM}$ and (b) $262 \mathrm{mM} \mathrm{EtOH}$ are normalized at their respective maxima. The lines are Lorentzian-Gaussian least squares fits.

\section{Fig. 8S.}

(a) Extrapolated conductivity $\kappa_{0}$ from the electron (open circles) in acetonitrile/isooctane solutions vs. the net molar concentration of $\mathrm{MeCN}\left(23{ }^{\circ} \mathrm{C}\right)$. The line drawn through the symbols is the optimum fit to eq. (6). (b) To the left: The same plot on the extended logarithmic scale; the solid line is a fit to $\left\langle\mu_{n}\right\rangle /\langle\mu\rangle=1+K_{e q} c+K_{n} c^{n}$ (see the text). To the right: ratio $r$ (open squares) for the same solution (50 ns delay time, $9 \times 10^{18}$ photon $/ \mathrm{cm}^{2}$ pulse of $1064 \mathrm{~nm}$ photons.)

\section{Fig. 9S.}

(a) Conductivity kinetics from acetonitrile/iso-octane solutions photoionized using 248 $\mathrm{nm}$ light (a thin line plotted to the left). Also shown are the $\Delta \kappa$ signals induced by the subsequent excitation of trapped electrons by $1064 \mathrm{~nm}$ light (cf. Fig. 3 in the text). The same excitation conditions were used as in Fig. $8 \mathrm{~S}(\mathrm{~b})$. The amplitude of $\Delta \kappa$ follows the decay kinetics of $\kappa-\kappa_{i} \approx \kappa$, so that the ratio $r$ is independent of the delay time. (b) $\kappa$ (to the left and to the bottom) and $\Delta \kappa$ (to the right and to the top) kinetics in room temperature Ar-saturated iso-octane solutions containing $\mathrm{MeCN}$ (the net solute concentrations are given in the plot). The smooth lines are exponential fits. The smooth lines drawn through the $\Delta \kappa$ data are Gaussian fits.

\section{Fig. 10S.}


Results similar to those in Fig. 9S (a) are shown for $17 \mathrm{mM} \mathrm{MeCN}$ in Ar-saturated $n$ hexane. The solution temperatures are indicated in (a). Conductivity kinetics (solid line in (a) and dashed lines in (b) are plotted to the right), $1064 \mathrm{~nm}$ photon induced kinetics are plotted to the left in (b). The green line in (b) indicates the delay time of the $1064 \mathrm{~nm}$ pulse (using the same excitation conditions as in Fig. 9S). The smooth lines drawn through the $\kappa$ data in (a) are exponential fits.

\section{Fig. 11S.}

Power dependencies of ratio $r$ at $t_{L} \approx 50 \mathrm{~ns}$ for several concentrations of acetonitrile in room temperature $n$-hexane (the concentrations and photon fluences are indicated in (b)). In the high concentration limit ( $K>>1$, see Fig. 4(a)) the initial slope approaches the product of photodetachment cross section and the lifetime of the $\left(e^{-}: \mathrm{MeCN}\right)_{\text {solv }}$ state. (b) The same plot after normalization at $8.5 \times 10^{18}$ photons $/ \mathrm{cm}^{2}$.

\section{Fig. 12S.}

Decay kinetics of photoconductivity for a $5 \mu \mathrm{m}$ solution of anthracene in $n$-hexane containing $12 \mathrm{mM} \mathrm{EtOH}$ (the temperatures are indicated in the plot). The signal is mainly from the electrons generated by 2-photon ionization of anthracene by $248 \mathrm{~nm}$ laser light. The smooth curves are exponential fits. Note that at the lowest temperature of $2{ }^{\circ} \mathrm{C}$ the kinetics becomes exponential only after $100 \mathrm{~ns}$. This delay is due to slow settling of the electron equilibria (see Figs. 9 and 10) at low temperature, in dilute alcohol solutions.

\section{Fig. 13S.}

Normalized conductivity signal $\kappa_{i}$ from ions (anions and cations) in ethanol $/ n$-hexane solutions vs. [EtOH] (i.e., the conductivity signals attained at the end of electron scavenging in the solution). The ions were generated by 2-photon ionization of room temperature Ar- and $\mathrm{SF}_{6}$-saturated solutions (filled diamonds and circles, respectively), Ar-saturated solution containing $0.3 \mathrm{mM}$ benzene as a sensitizer (filled squares), and $\mathrm{SF}_{6}$ saturated $5 \mu \mathrm{m}$ anthracene solutions at $2{ }^{\circ} \mathrm{C}$ (filled triangles), $8{ }^{\circ} \mathrm{C}$ (open squares) and 15 ${ }^{\circ} \mathrm{C}$ (filled downward triangles). Also shown (to the right) is the mobility of $\mathrm{F}^{-}$(see Fig. $14 \mathrm{~S}(\mathrm{~b}))$. Addition of alcohol reduces anion mobility causing the decrease in the conductivity signal from the ions. The line is a guide for the eye.

\section{Fig. 14S.}

(a) Time-of-flight conductivity traces (the electrode spacing is $0.8 \mathrm{~mm}$, the voltage is 5 $\mathrm{kV}$ ) from $0.65 \mathrm{mM}$ triethylamine in $\mathrm{SF}_{6}$-saturated $n$-hexane $\left(23{ }^{\circ} \mathrm{C}\right)$ as a function of 
ethanol concentration (molar concentrations are indicated in the plot). $\mathrm{SF}_{6}$ rapidly scavenges the electron within the duration of the $248 \mathrm{~nm}$ pulse yielding a fluoride anion, $\mathrm{F}^{-}$. A $100 \mu \mathrm{m}$ slit is placed near the cathode so that the conductivity signal is from migrating $\mathrm{F}^{-}$anions (the conductivity at the flat region is ca. $100 \mathrm{pS} / \mathrm{cm}$ ). The cations rapidly migrate in the electric field and discharge at the cathode; a fraction of these cations decay via. neutralization in the bulk (this reaction is over in $0.5 \mathrm{~ms}$ ). The higher the alcohol concentration, the longer is the time of flight for the anions. (b) The plot of drift mobility for $\mathrm{F}^{-}$vs. [EtOH]. See also Fig. $13 \mathrm{~S}$.

\section{Fig. 15S.}

First order constant $\Delta k$ for electron decay in room temperature, Ar-saturated solutions of (a) ethanol and (b) methanol in $n$-hexane vs. the molar concentration (to the bottom) or mole fraction (to the top) of these two alcohols. Filled symbols are for protiated, open symbols are for deuterated alcohols. See section 4.2.2 for more detail.

\section{Fig. 16S.}

Dependencies of $1064 \mathrm{~nm}$ photon induced conductivity signals from very dilute $(<5$ $\mathrm{mM}$ ) ethanol $/ n$-hexane solutions at (a) $2{ }^{\circ} \mathrm{C}$ and (b) $23{ }^{\circ} \mathrm{C}$ vs. the delay time $t_{L}$ of the IR pulse (to the right). The decay kinetics of conductivity signals from the electron (plotted to the left) are juxtaposed for comparison. At both temperatures, the amplitudes of the $\Delta \kappa$ signals decrease in proportion to $\kappa_{e}\left(t_{L}\right)=\kappa-\kappa_{i}$, suggesting no trapping by higher alcohol clusters in the course of the slow "scavenging" reaction (Fig. 15S). In this plot and in Figs. 17S(b) and 18S, the fluence of $1064 \mathrm{~nm}$ photons is $9 \times 10^{18}$ photon $/ \mathrm{cm}^{2}$.

\section{Fig. 17S.}

Decay kinetics of (a) $248 \mathrm{~nm}$ photon induced conductivity signals and (b) $1064 \mathrm{~nm}$ induced signals (for $t_{L}=70 \mathrm{~ns}$ ) from Ar-saturated $n$-hexane solutions containing $6 \mathrm{mM}$ $\mathrm{EtOH}$. The temperatures of the solutions are indicated in the plot. The smooth curves in (a) are exponential fits; the smooth curves drawn through the symbols in (b) are weighted sums of a Gaussian (with the time profile of the excitation pulse) and the same Gaussian convoluted with an exponential function. To facilitate the comparison, $\Delta \kappa$ kinetics are vertically spaced.

\section{Fig. 18S.}

Same as Fig. $17 \mathrm{~S}(\mathrm{~b})$ : the concentration dependence of $\Delta \kappa$ kinetics at $14.9{ }^{\circ} \mathrm{C}$. Ethanol concentrations are indicated in the plot. 


\section{Fig. 19S.}

Power plot for the ratio $r$ (compare with Fig. 11S(a)) for several concentrations of EtOH in room temperature, Ar-saturated $n$-hexane. The net solute concentrations are indicated in the plot. Note the logarithmic vertical scale.

\section{Fig. 20S.}

(a) The dependence of parameter $K=\left\langle\mu_{n}\right\rangle /\langle\mu\rangle-1$ on [EtOH] for Ar-saturated $n$-hexane at $23{ }^{\circ} \mathrm{C}$ (circles). The solid line is a $K \propto c^{4}$ plot. (b) The dependence of ratio $r$ (at $t_{L} \approx 50 \mathrm{~ns}$ ) vs. $K$ for ethanol in $n$-hexane for fluences $J=8.1 \times 10^{18}$ (circles) and $5.4 \times 10^{17}$ photon $/ \mathrm{cm}^{2}$ of $1064 \mathrm{~nm}$ light (squares); the same series are shown in (a). The lines are guides for the eye. Compare with Fig. 4(b). The open and filled symbols (b) correspond to different integration methods for the $\Delta \kappa$ signal.

\section{Fig. 21S.}

(a) Plots of ratio $r$ vs. fluence $J$ of $1064 \mathrm{~nm}$ photons for simulation parameters given in the Appendix. (b) Same plot, after normalization at $J=8 \times 10^{18}$ photon $/ \mathrm{cm}^{2}$. The constant $K$ is indicated in plot (a). Compare with Figs. 11S and 19S.

\section{Fig. 22S.}

The same calculation as in Fig. 21S: the ratio $r$ is plotted vs. $K$ for different fluences of $1064 \mathrm{~nm}$ photons (indicated in the plot). Compare with Figs. 4 and 20S. 


\section{$S_{n}$ species in EtOH/ $n$-hexane}
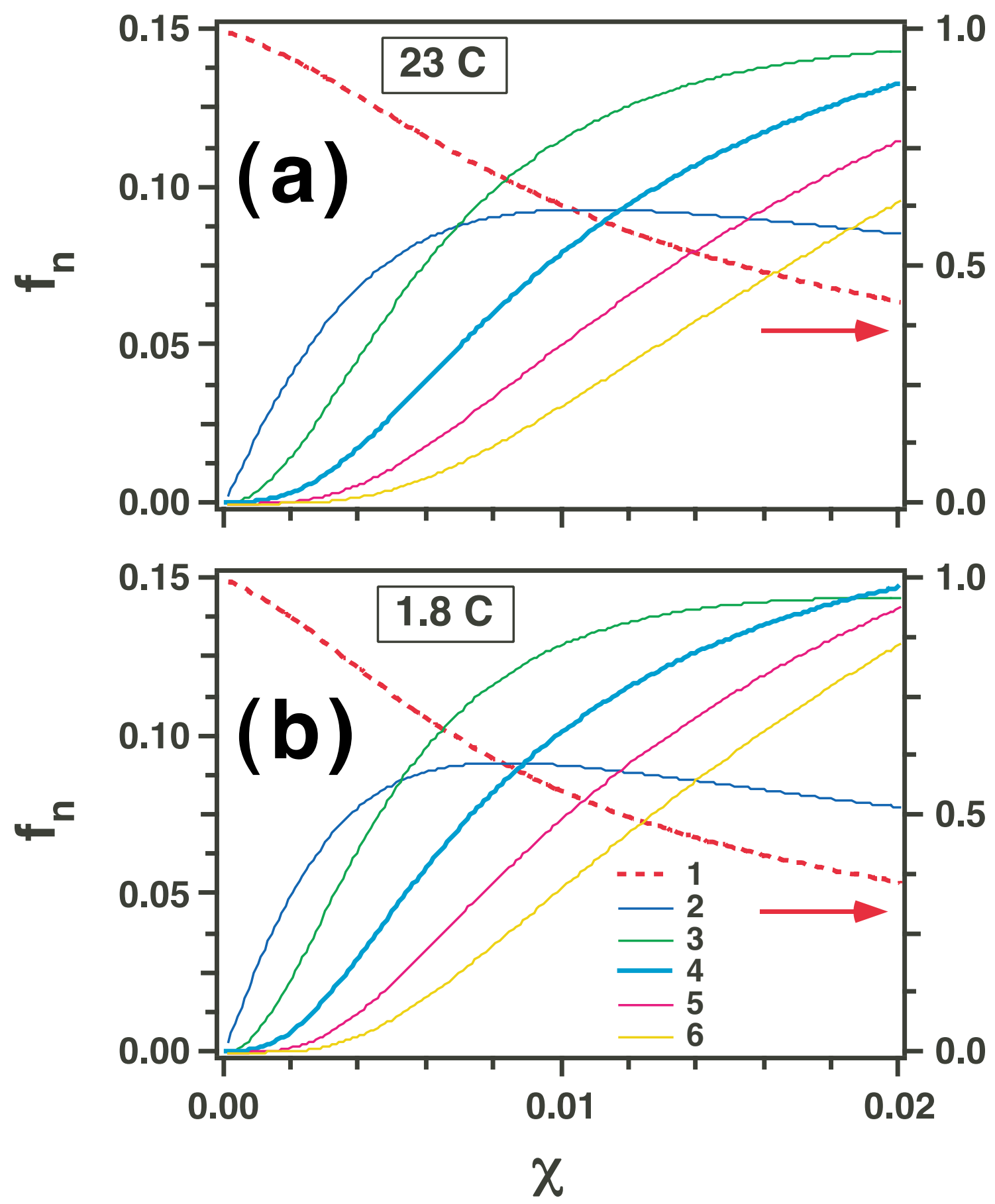

Figure 1S; Shkrob \& Sauer 

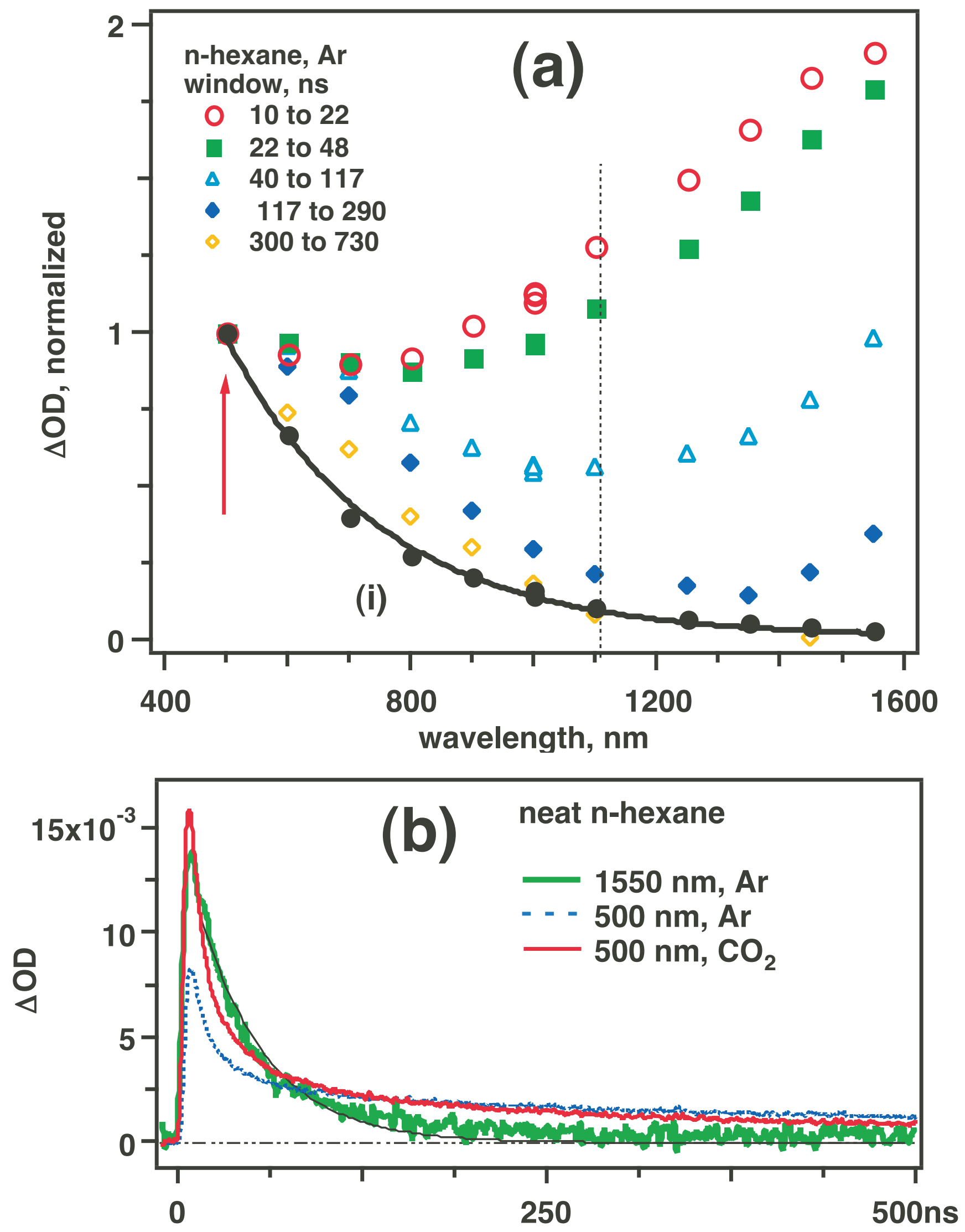

Figure 2S; Shkrob \& Sauer time 

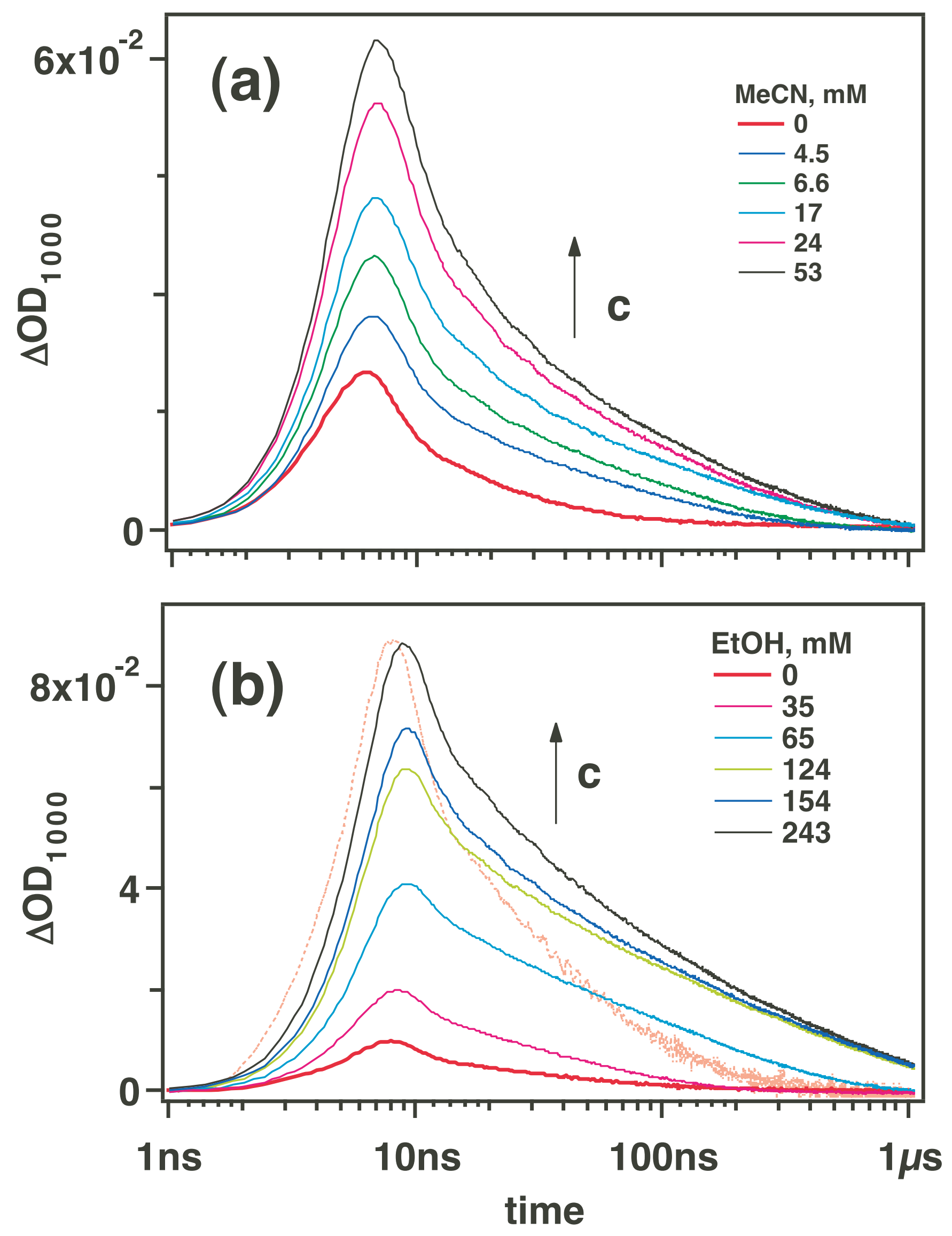

Figure 3S; Shkrob \& Sauer 
TA spectra in $\mathrm{MeCN} / \mathrm{n}$-hexane

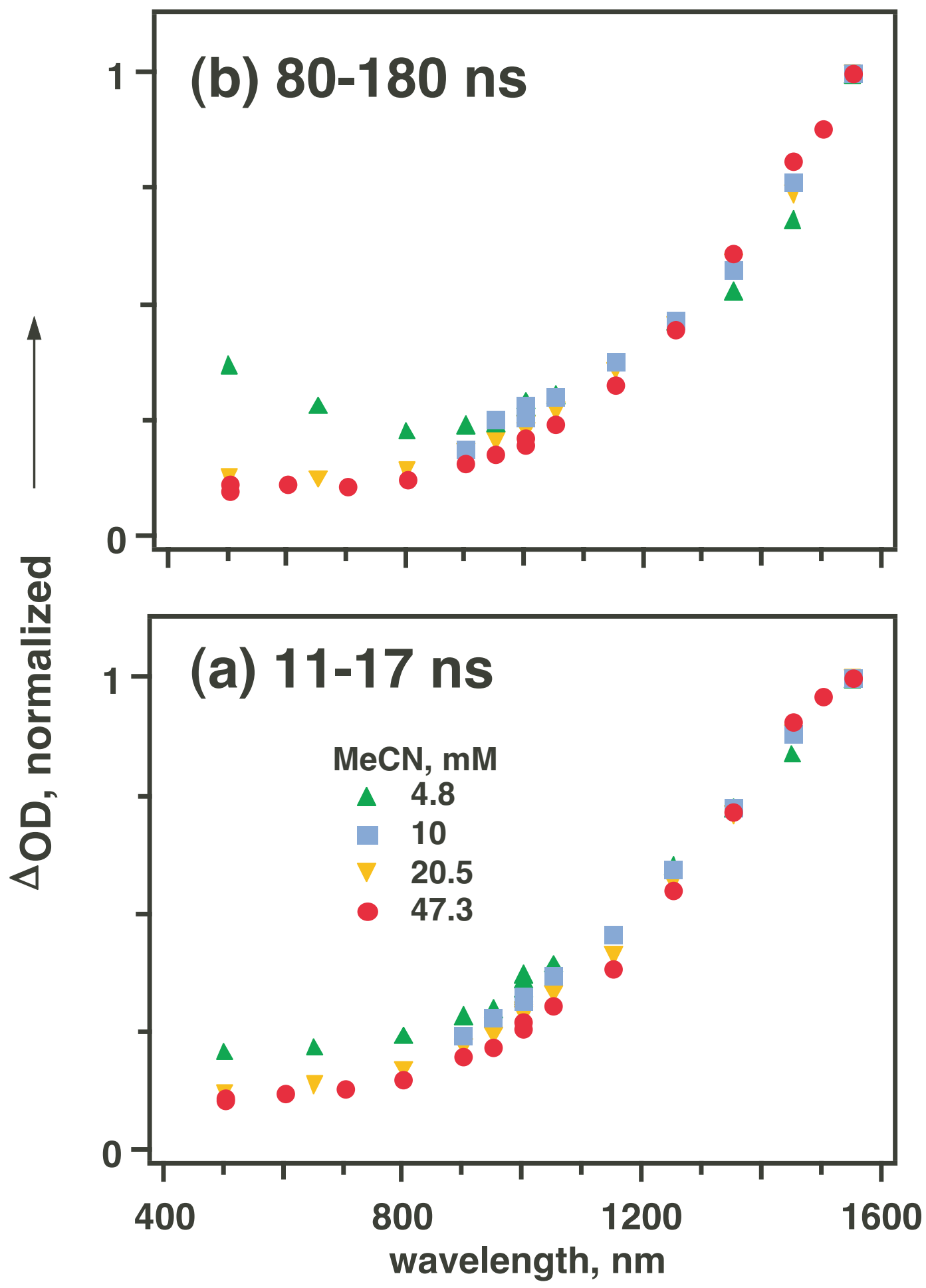

Figure 4S; Shkrob \& Sauer 

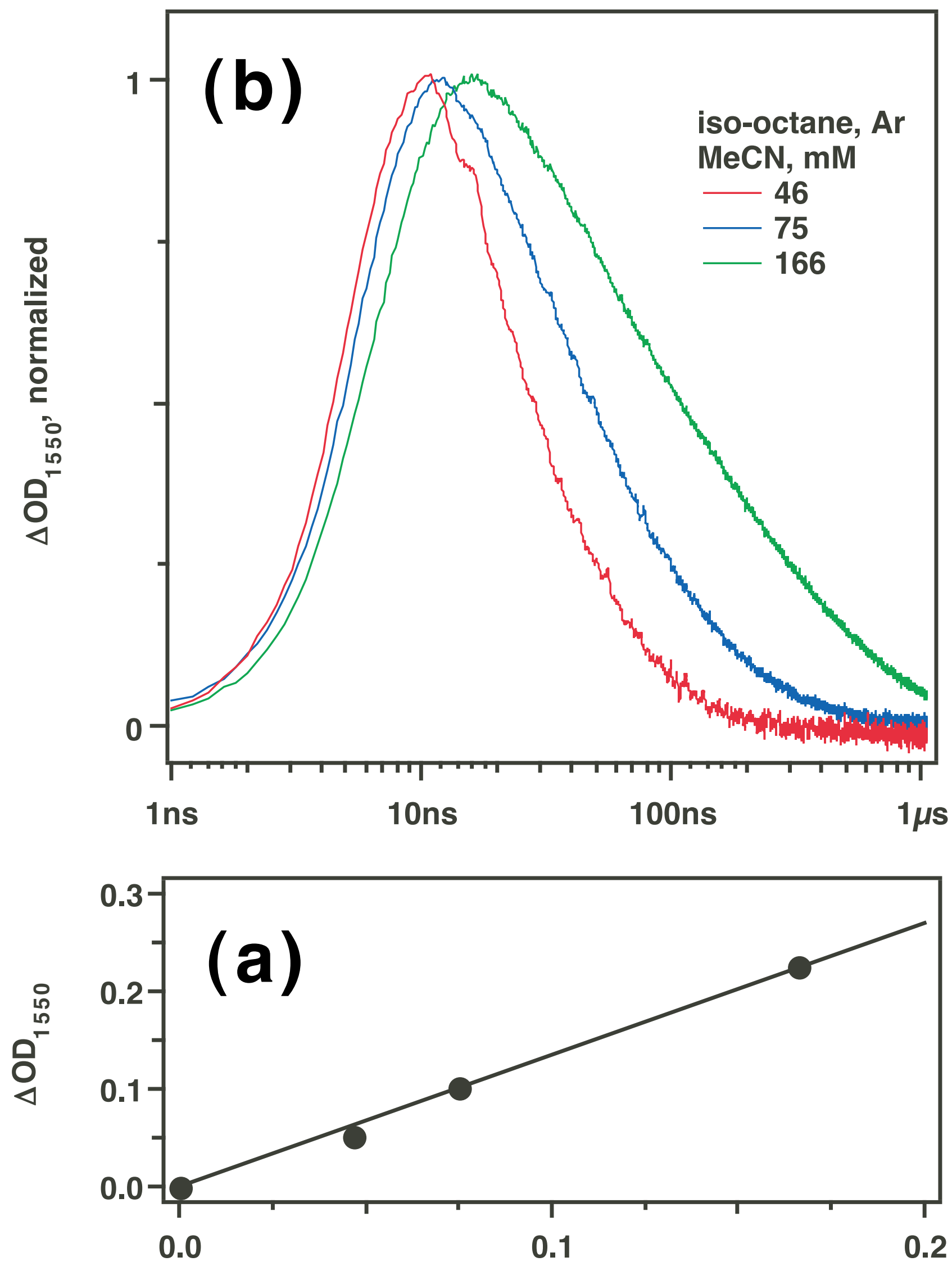

Figure 5S; Shkrob \& Sauer [MeCN], M 
TA spectra in $\mathrm{MeCN}$ / iso-octane

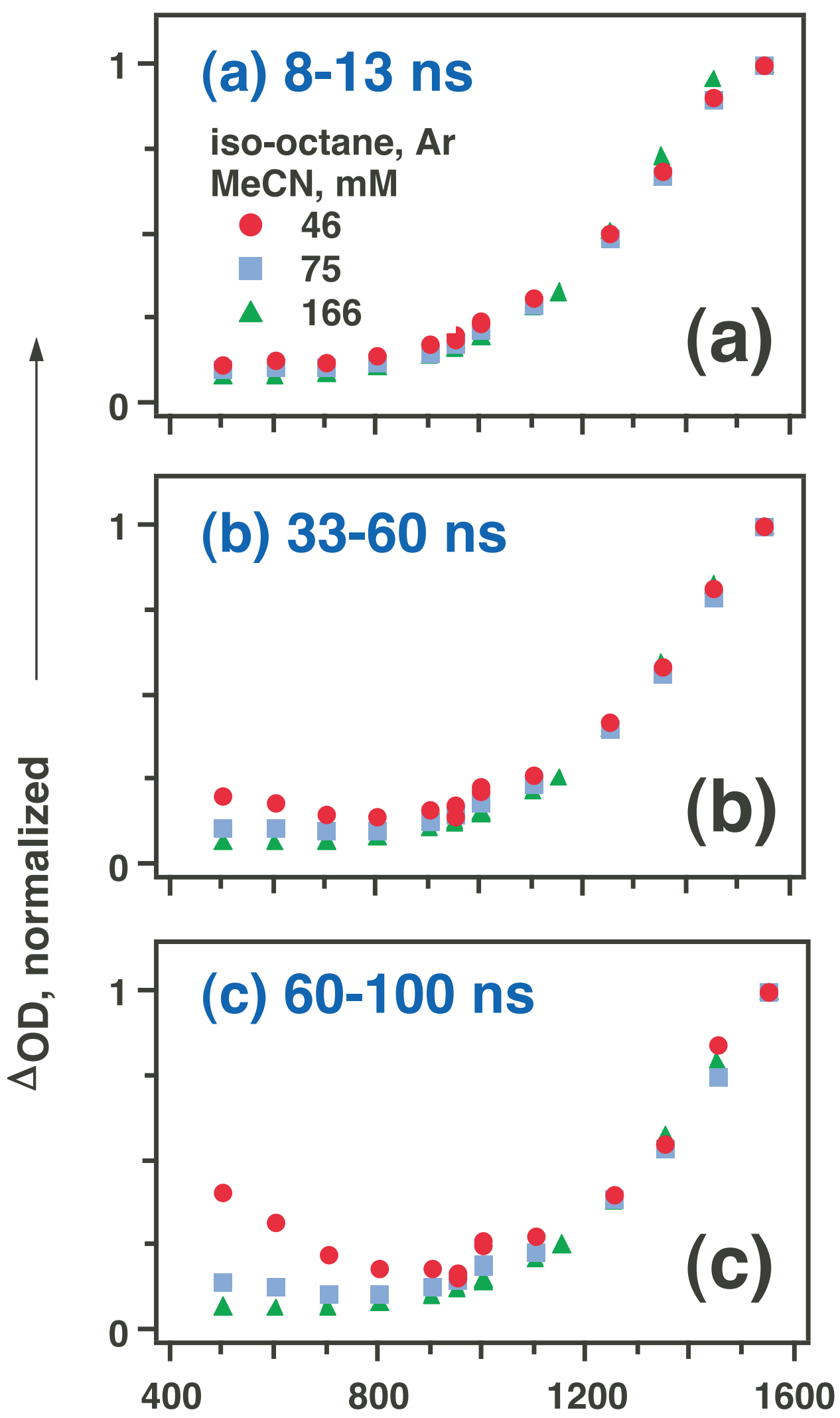

:igure 6S; Shkrob \& Sauer 

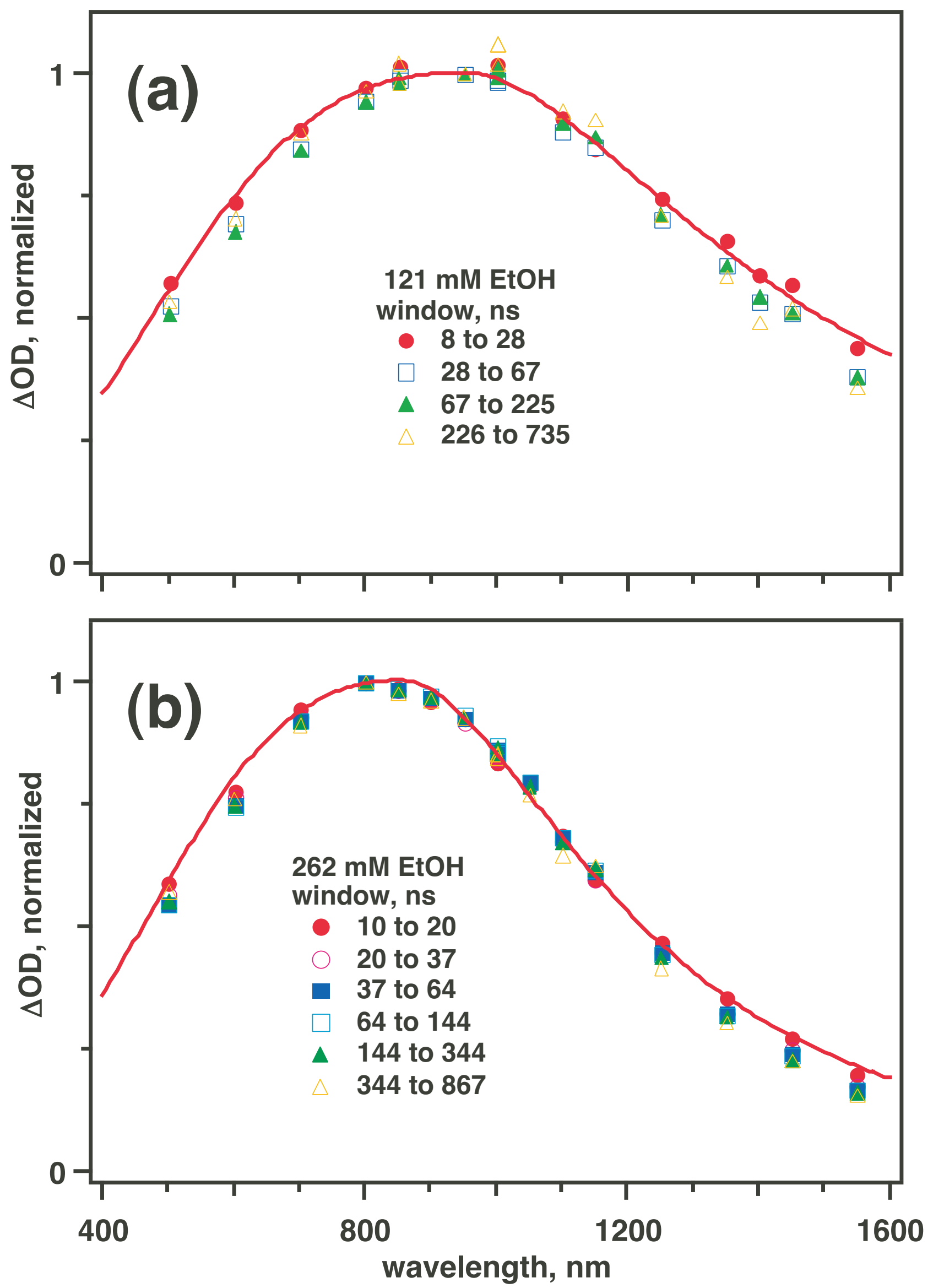

Figure 7S; Shkrob \& Sauer 

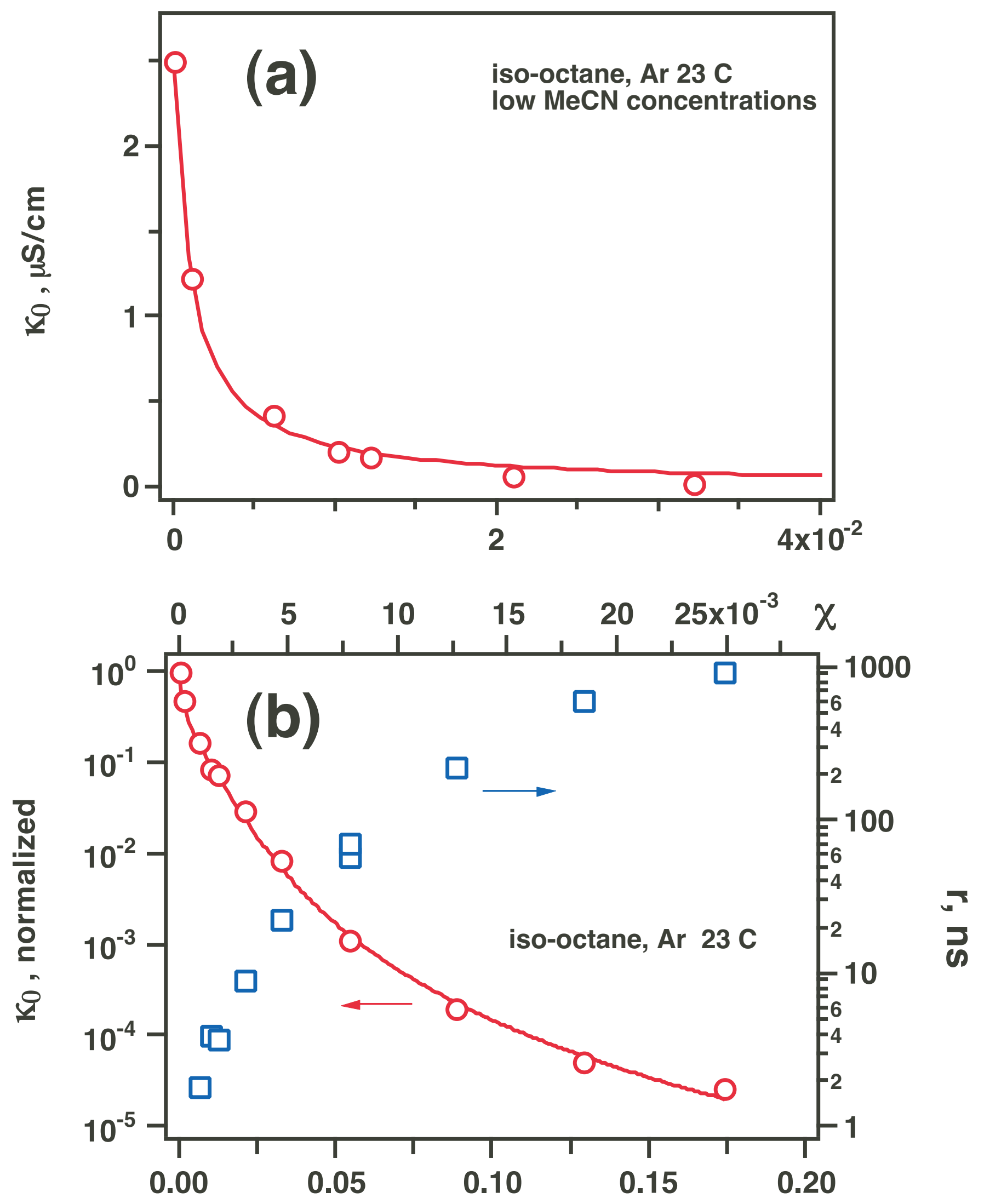

Figure 8S; Shkrob \& Sauer [MeCN], M 

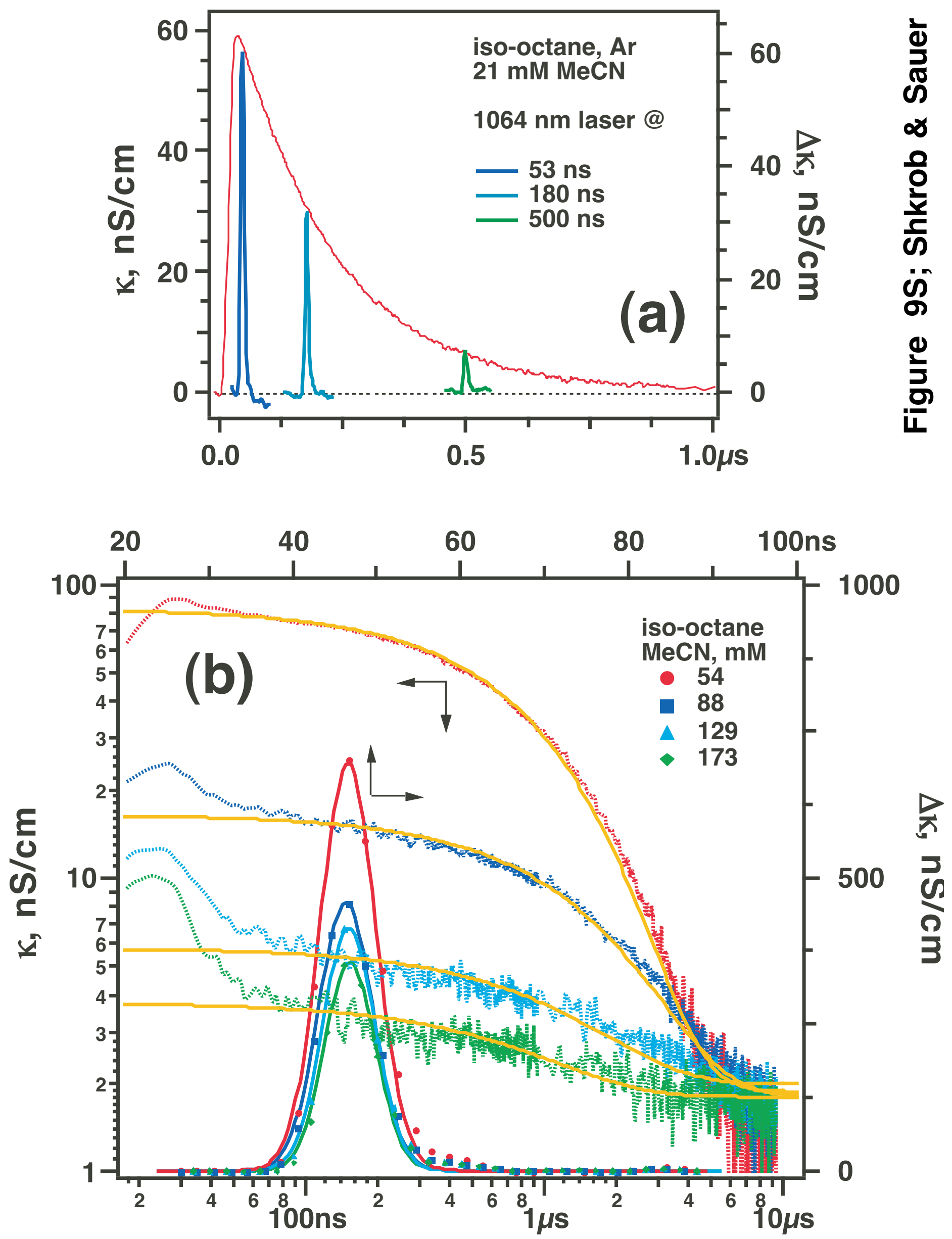

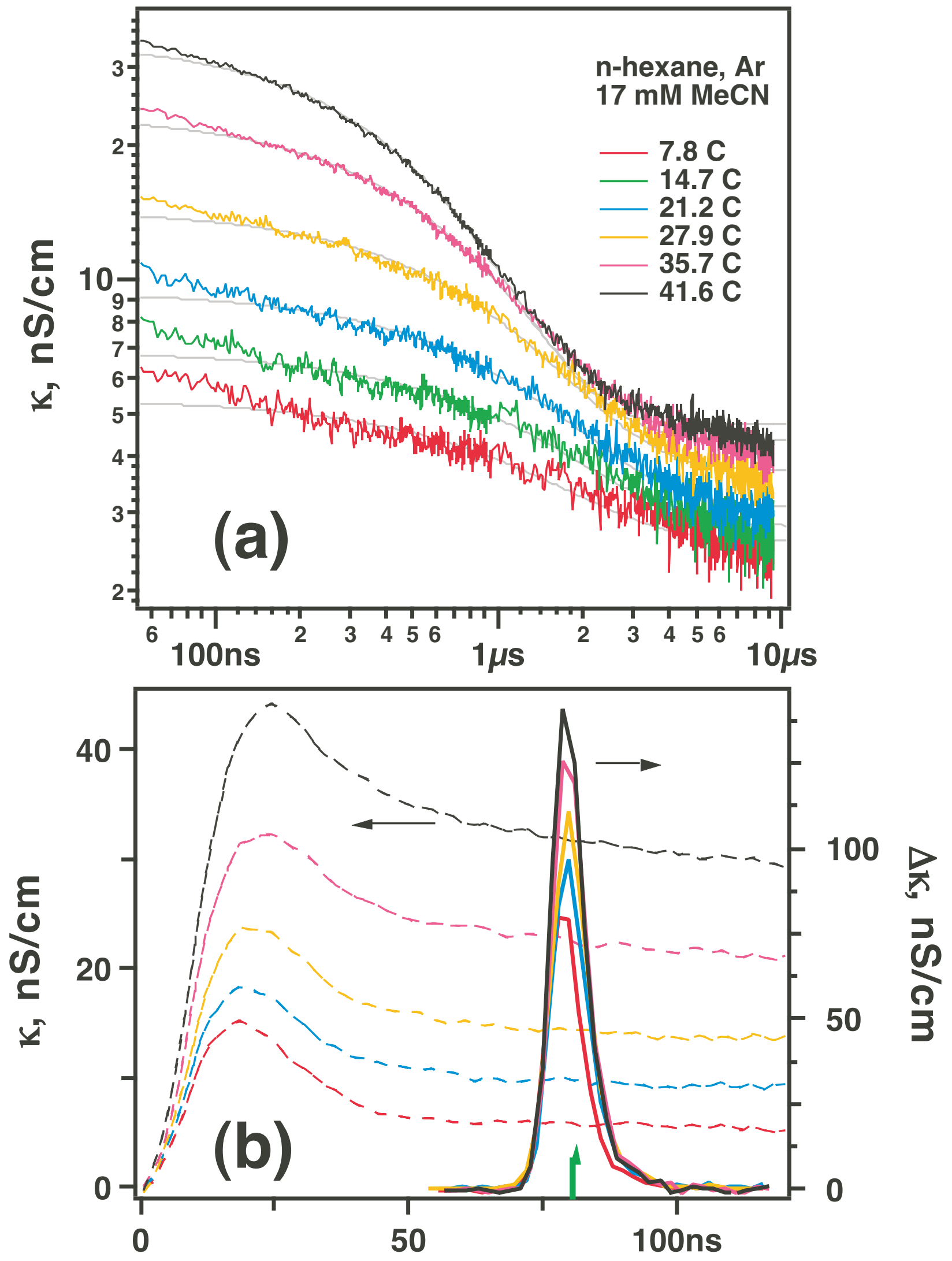

Figure 10S; Shkrob \& Sauer 

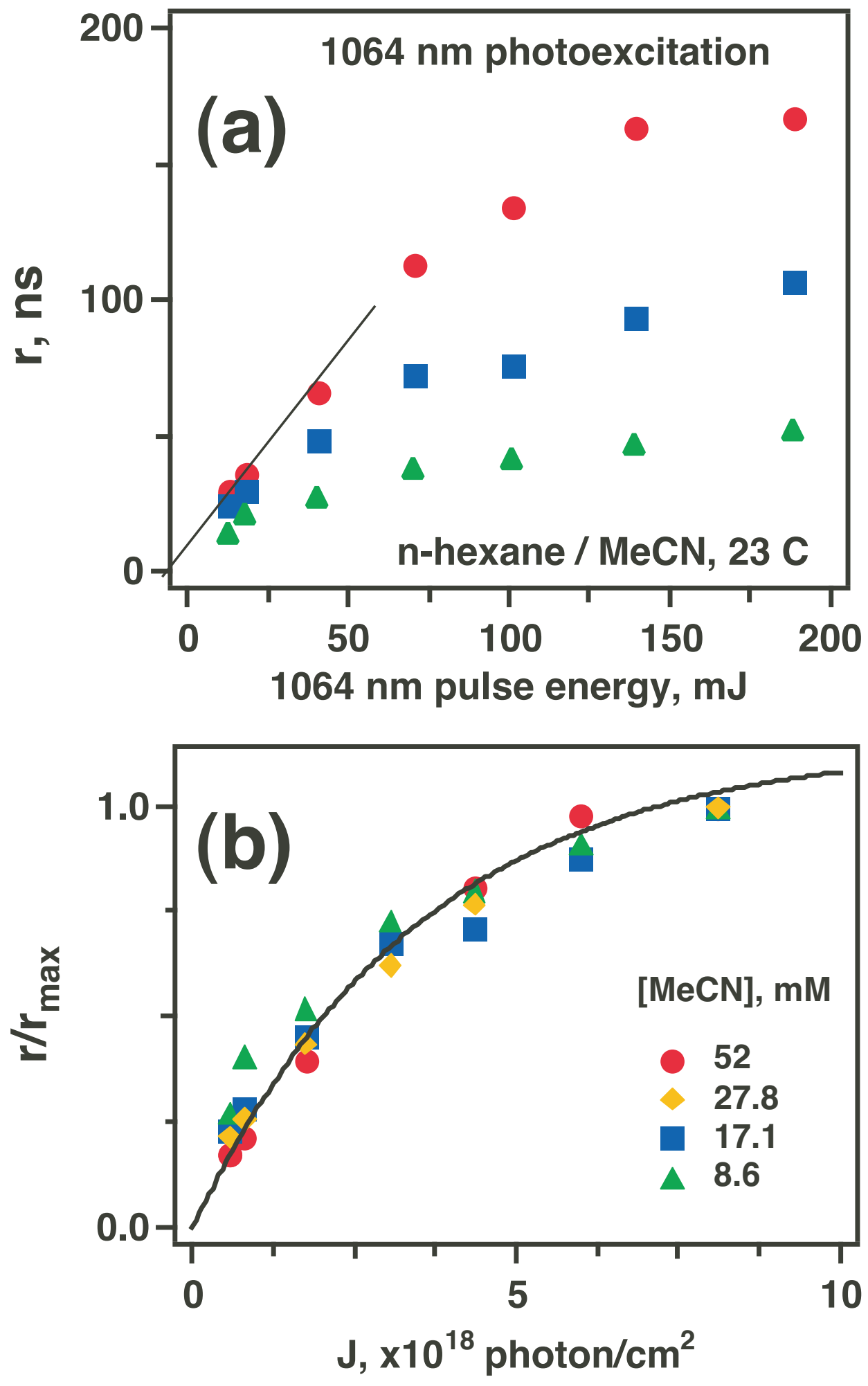

Figure 11S; Shkrob \& Sauer 


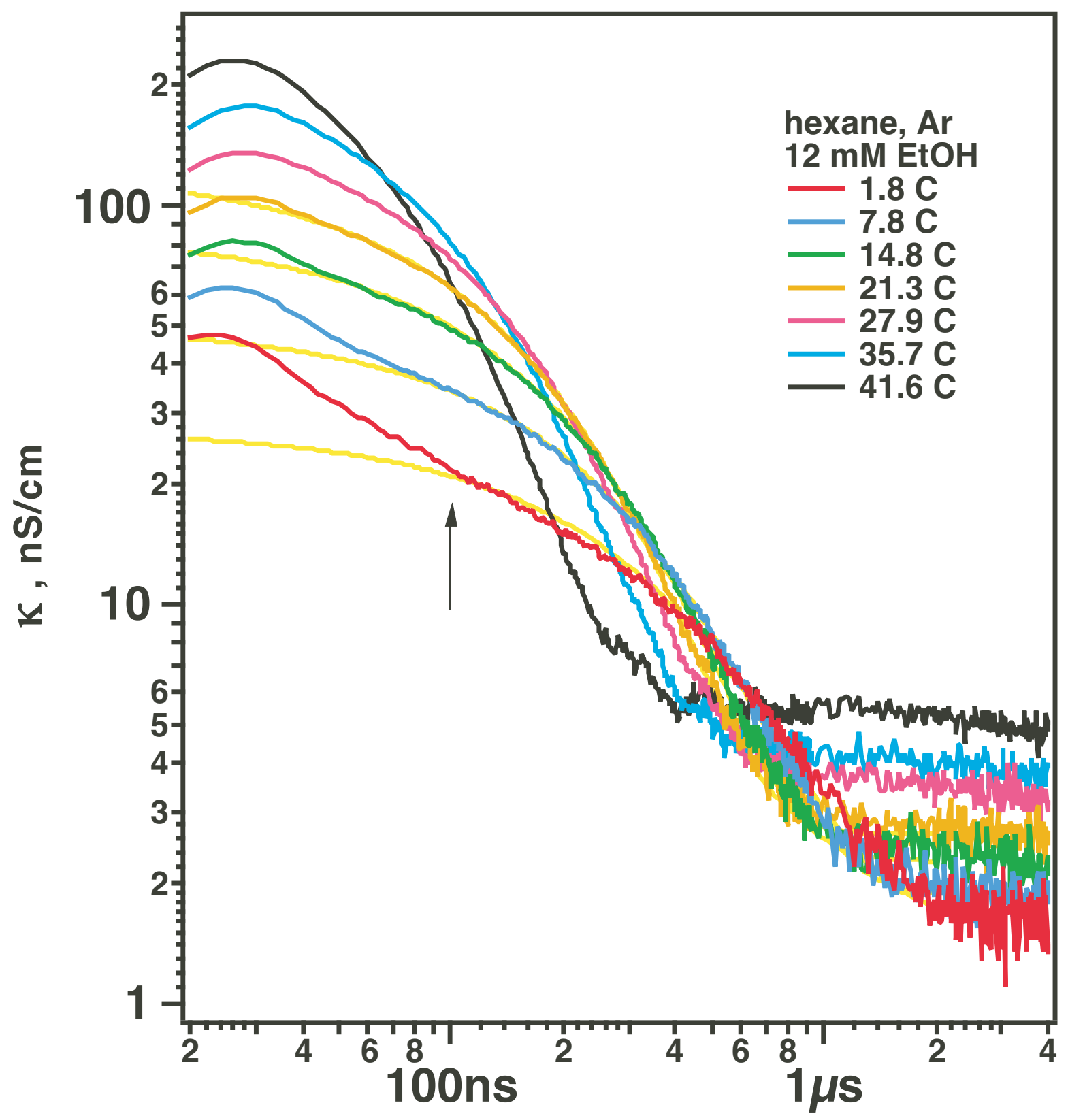

Figure 12S; Shkrob \& Sauer 


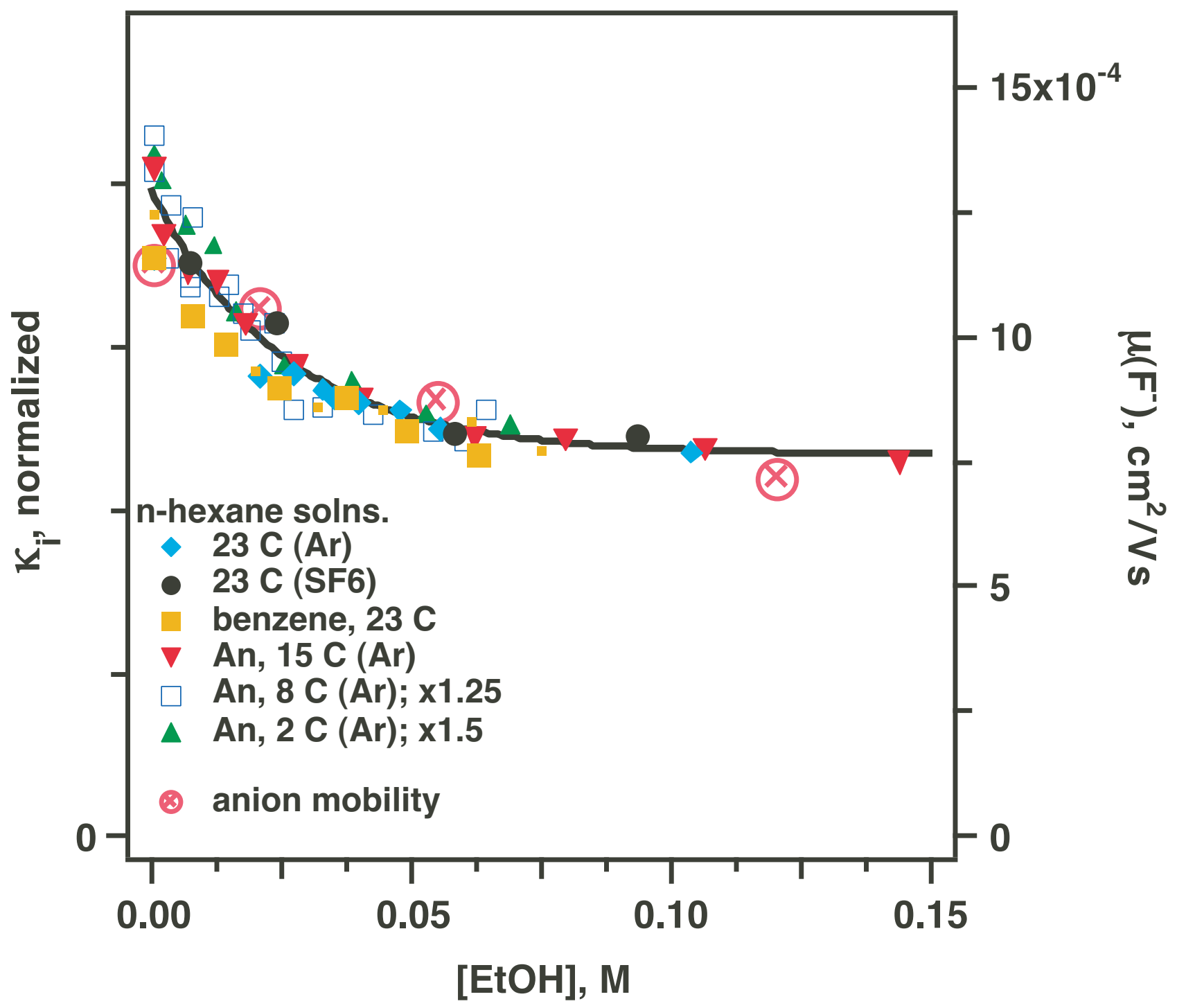

Figure 13S; Shkrob \& Sauer 


\section{TOF \& anion mobility for EtOH/n-hexane}
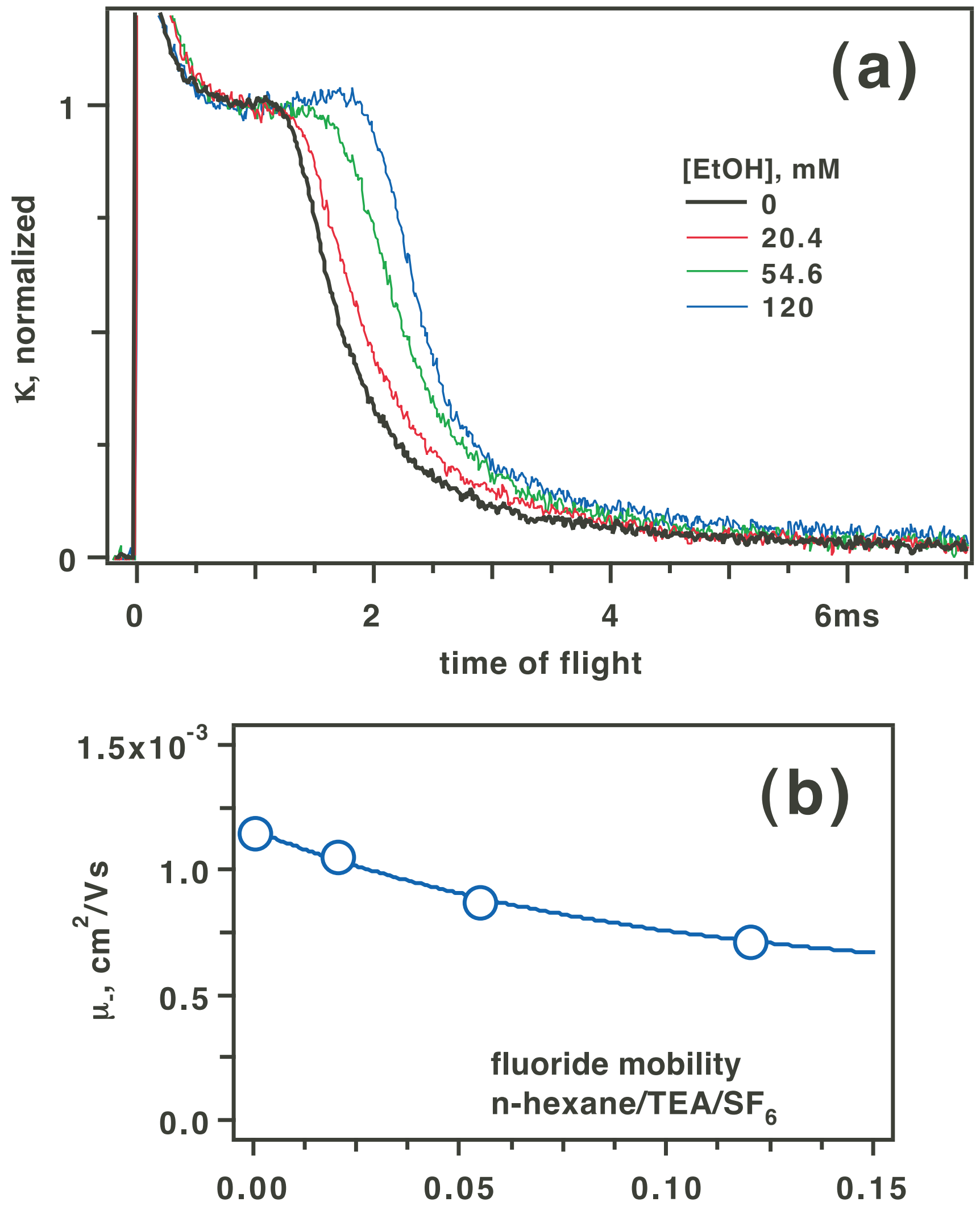

تigure 14S; Shkrob \& Sauer [EtOH], M 

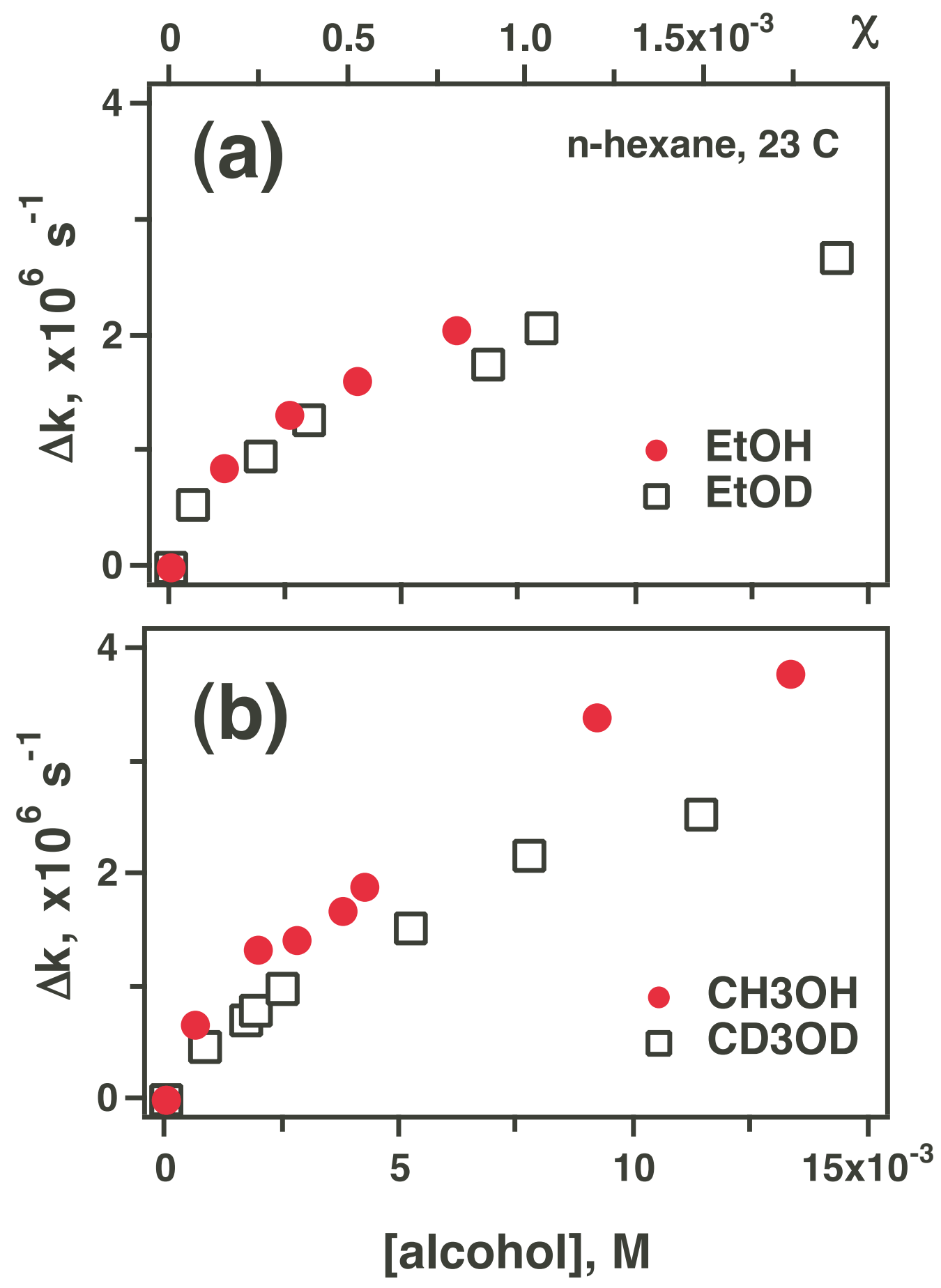

Figure 15S; Shkrob \& Sauer 

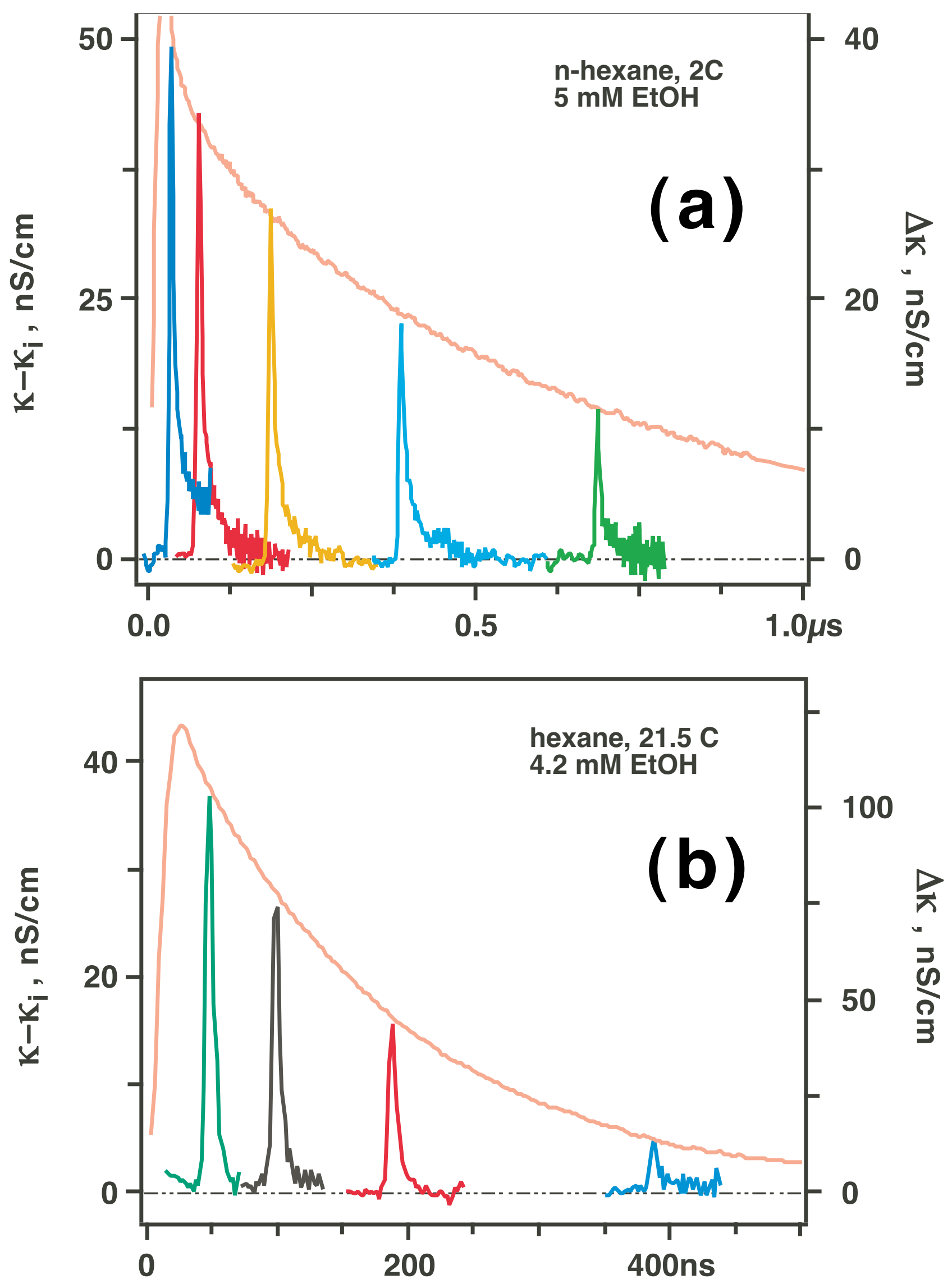

Figure 16S; Shkrob \& Sauer 


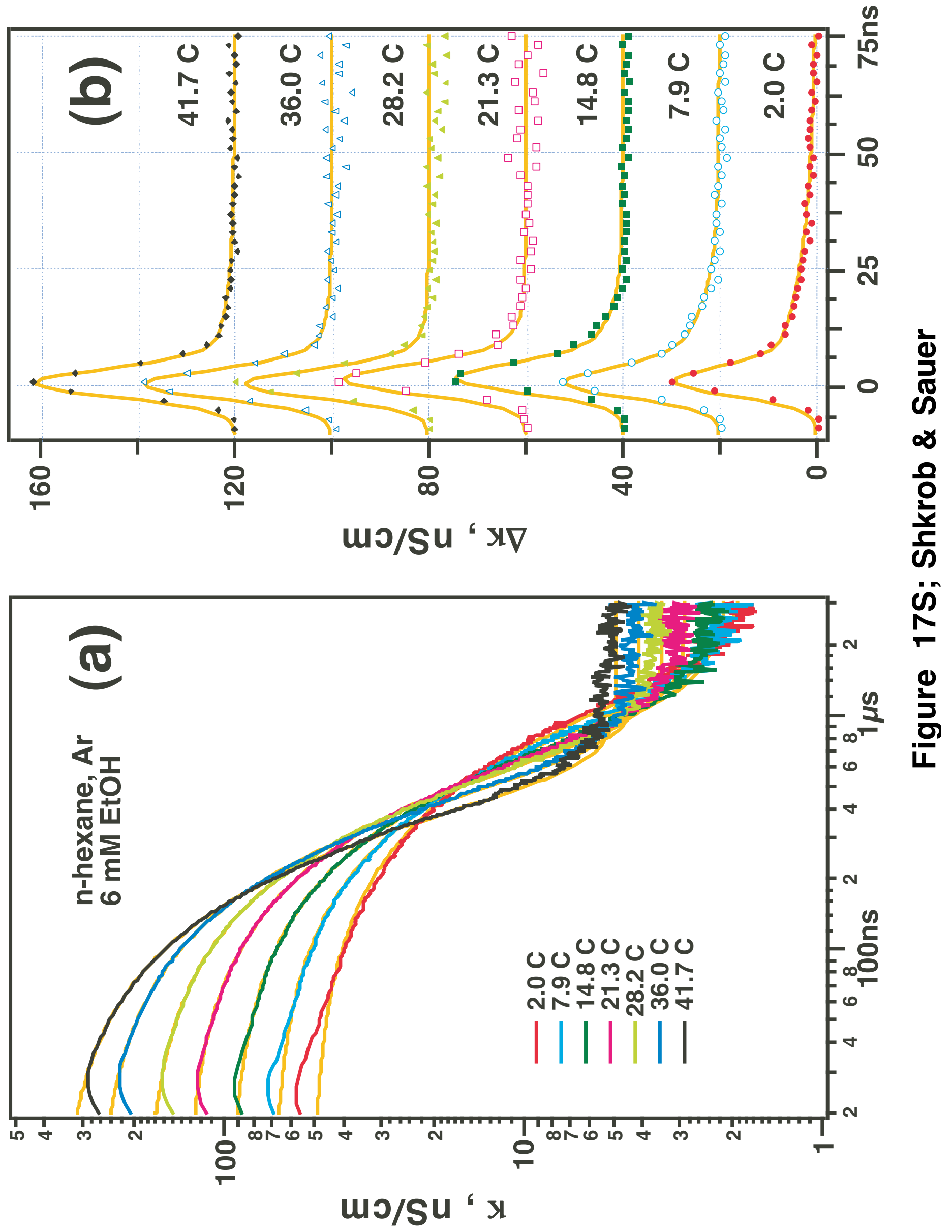




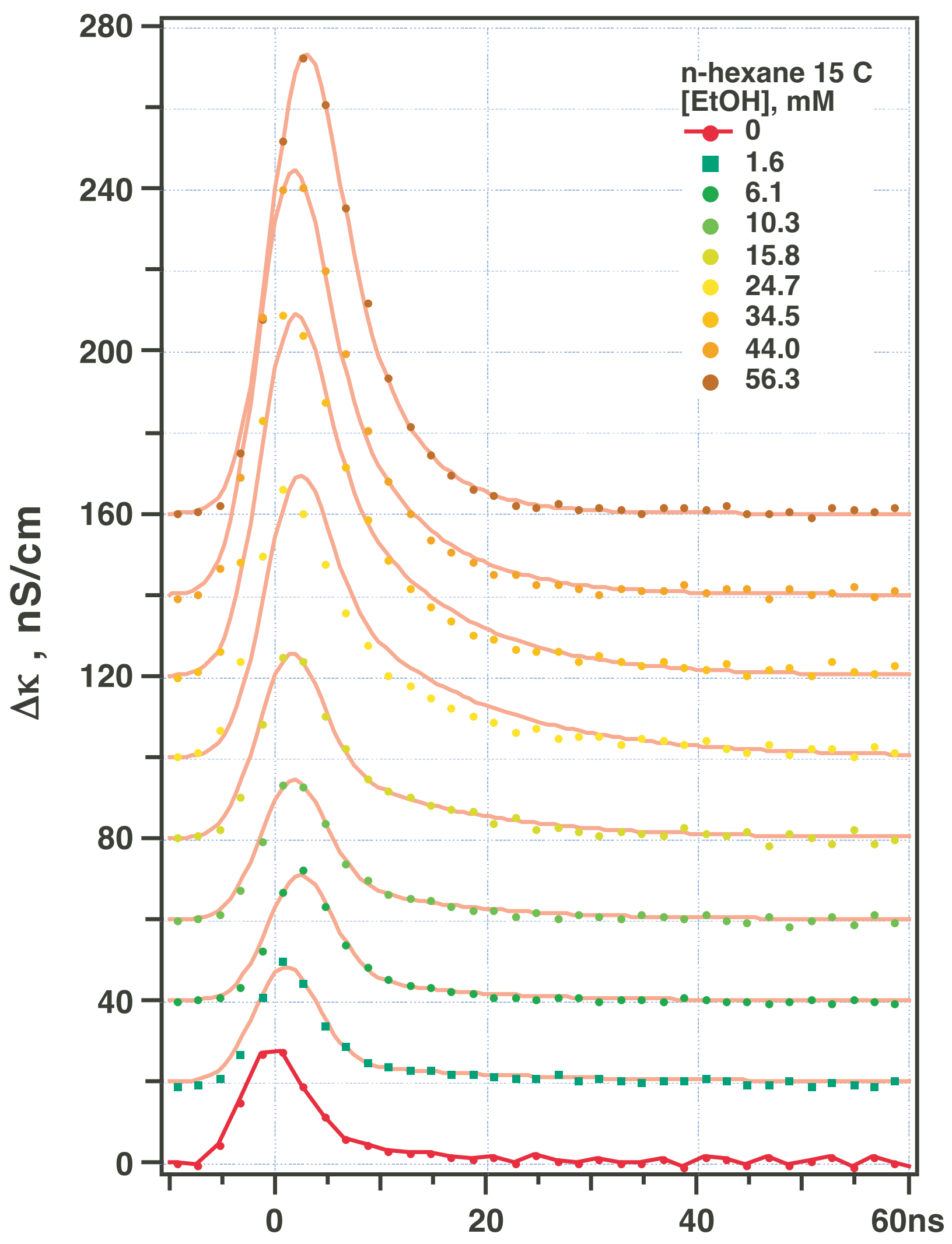

Figure 18S; Shkrob \& Sauer 


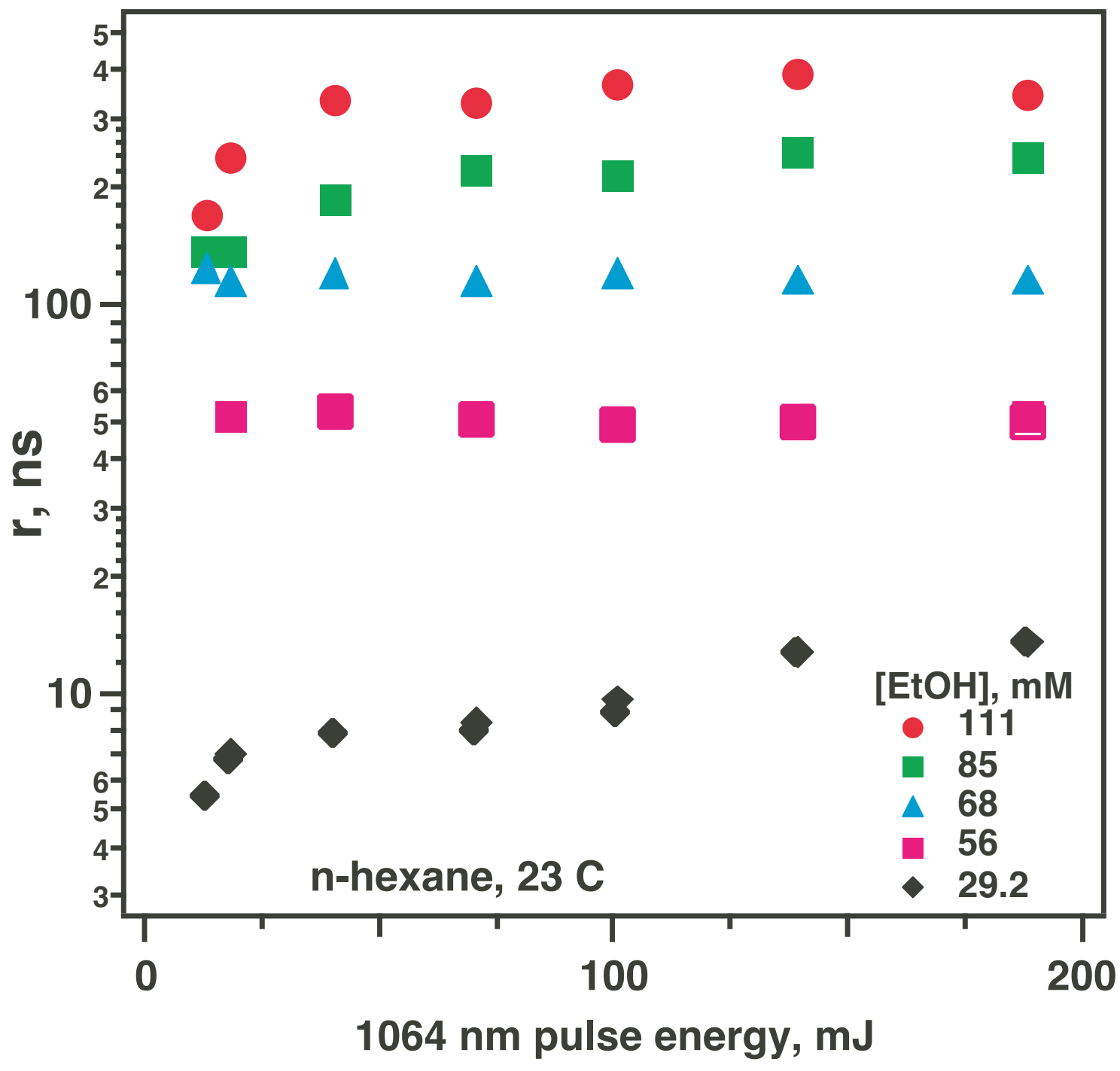

Figure 19S; Shkrob \& Sauer 

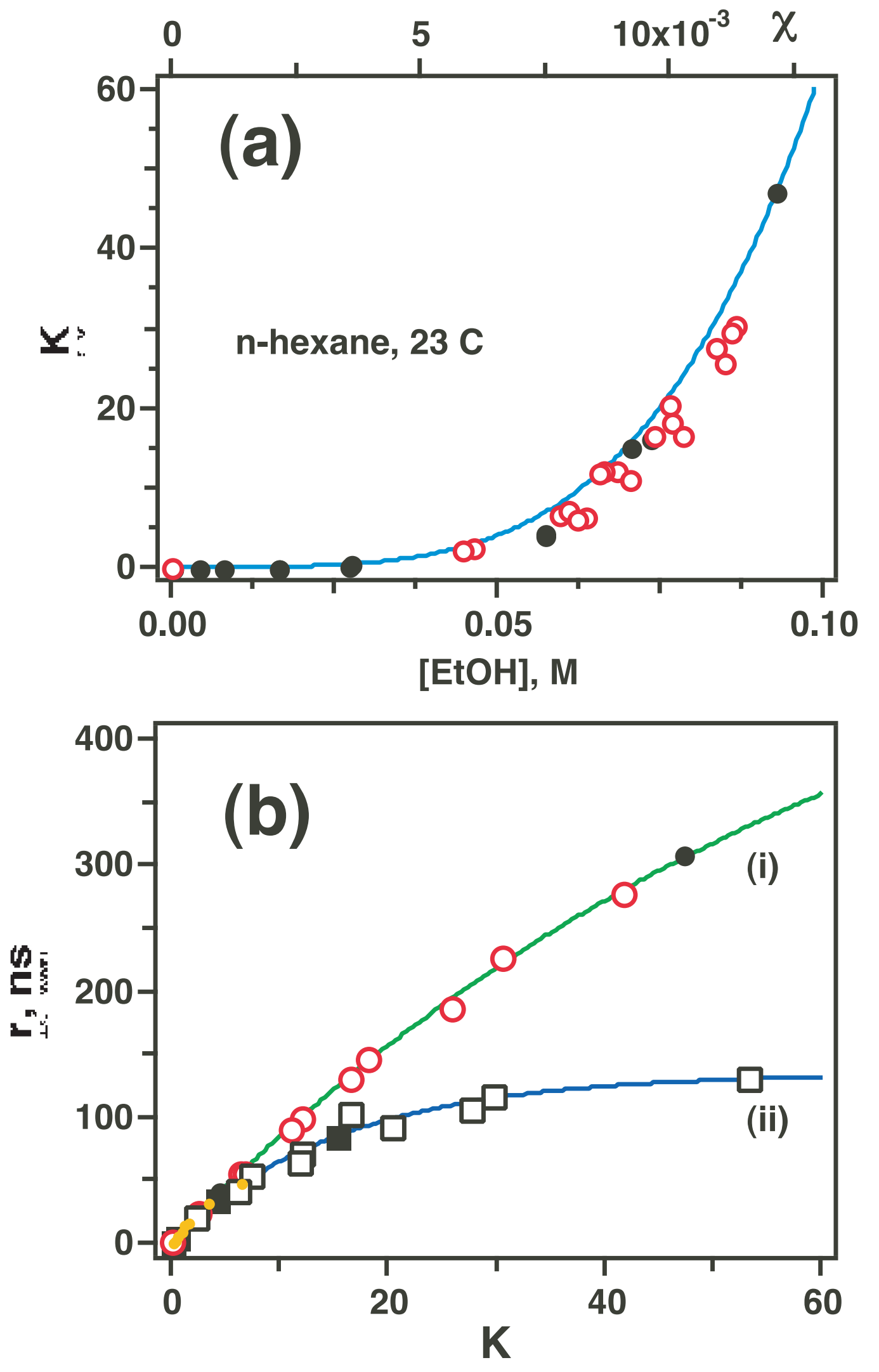

Figure 20S; Shkrob \& Sauer 

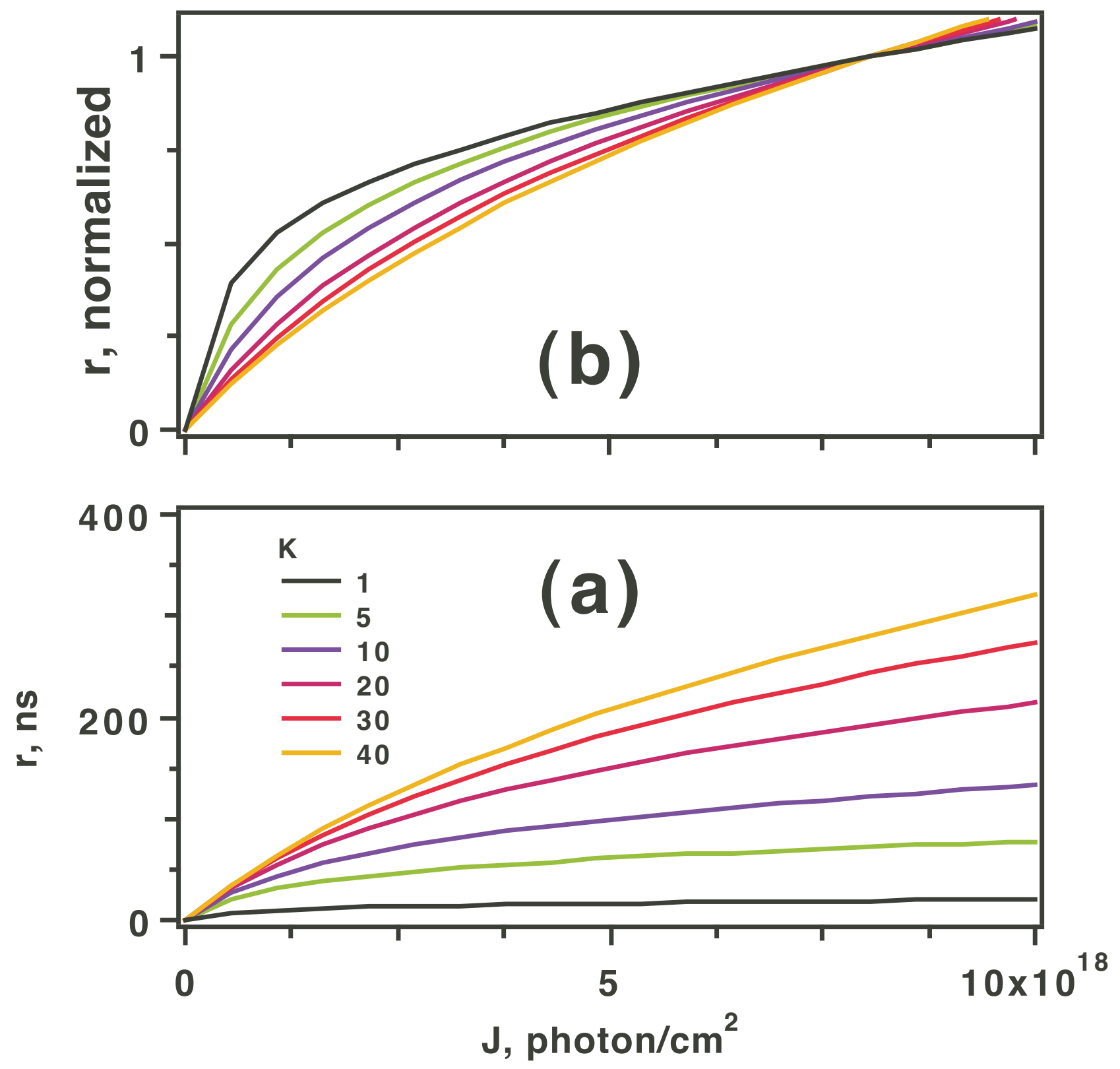

Figure 21S; Shkrob \& Sauer 


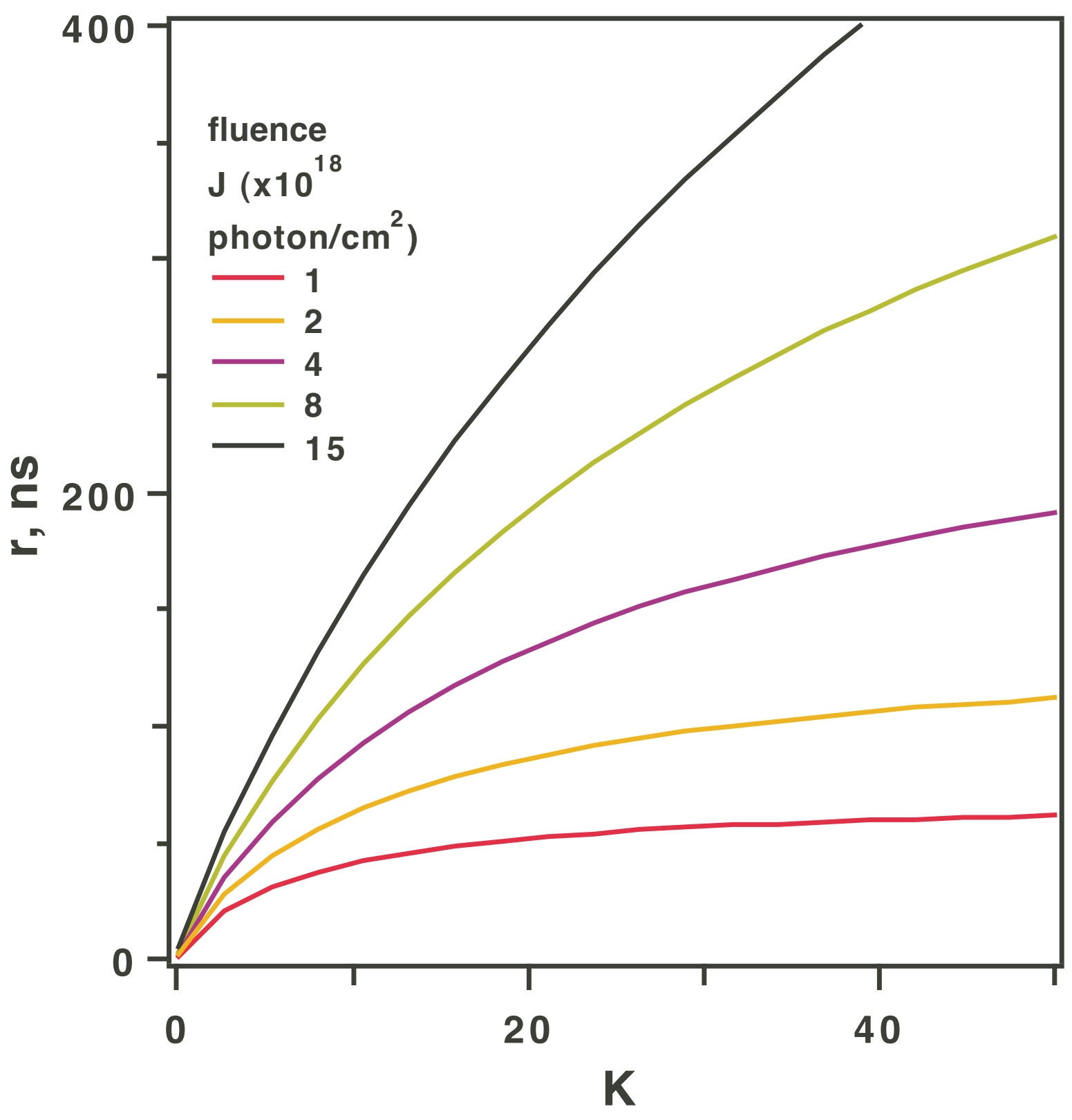

Figure 22S; Shkrob \& Sauer 